\title{
Alternative Design Procedure for RC-Braced Long Columns Based on New Moment Magnifiers Matrix
}

\author{
Mohamed Farouk $\mathbb{D},{ }^{1}$ Majed Alzara $\mathbb{D}^{\circ},{ }^{2}$ A. Ehab, ${ }^{3}$ and A. M. Yosri $\mathbb{i}{ }^{1,2}$ \\ ${ }^{1}$ Civil Engineering Department, Faculty of Engineering, Delta University for Science and Technology, Belkas, Egypt \\ ${ }^{2}$ Department of Civil Engineering, College of Engineering, Jouf University, Sakakah, Saudi Arabia \\ ${ }^{3}$ Department of Civil Engineering, Badr University in Cairo, Badr City, Egypt
}

Correspondence should be addressed to Majed Alzara; majedzara@ju.edu.sa

Received 9 March 2021; Revised 31 July 2021; Accepted 16 August 2021; Published 21 October 2021

Academic Editor: Giovanni Minafò

Copyright (c) 2021 Mohamed Farouk et al. This is an open access article distributed under the Creative Commons Attribution License, which permits unrestricted use, distribution, and reproduction in any medium, provided the original work is properly cited.

\begin{abstract}
Based on modified methods for the results of first-order analysis of RC columns, different codes approximate the second-order effects by using equations focusing on the maximum additional moment through the column height. These equations did not refer to the additional moments between the column and the connected beam, only the effect of the connected beams is taken into consideration by dealing with the effective length of the column, not the total length. Moreover, these equations did not take into account the second-order effect, which is caused by axial force and the inverse moments due to beam restriction for the column ends. This paper presents a new moment magnifiers matrix for the additional moments at the connection between braced columns and the connected beams as a simplified computation that can be used in the design procedure. That is through an equation based on transforming the original long column in second-order analysis to an equivalent isolated column. The equivalent column was represented as an element restricted with rotational spring support at its ends, and it is subjected to lateral distributed loads that have the same influence of the second-order effect on the induced additional moments in the long column. The suggested equivalent column can be used to form the additional bending moment diagram, also to compute the additional deformations as well. Numerous factors were analyzed linearly by using the presented new moment magnifiers matrix and finite element method, and the results proved the efficiency of the proposed model. Although the presented suggested model is based on the isolated analysis of the long column, the effect of the additional moments in the adjacent long column can be considered by presented two suggestions to improve the model. Also, development was proceeded on the model by modifying the flexural rigidity (EI) which is recommended in ACI to appropriate the time of failure. The additional moment values of the developed model were close to the values calculated by the ACI equation.
\end{abstract}

\section{Introduction}

In braced long columns, a column is subjected to axial load and equal or unequal end moments, which are caused by the connected beam loads, and deformed laterally due to the existence of end moments. The axial load and occurred lateral deflection cause additional bending moments along the column height which is called second order. The additional bending moments cause additional lateral displacements and rotations of the column and additional rotation of the members connecting into the column.
This, in turn, leads to change to the first-order bending moments through the column height and at the connected ends of the column with the beams, which are computed from an elastic frame analysis. The equations of equilibrium in a first-order analysis are derived by assuming that the deflections have a negligible impact on the internal forces in the members. In a second-order analysis, the equations of equilibrium consider the deformed shape of the structure. Instability can be investigated only via a second-order analysis because it is the loss of equilibrium of the deformed structure that causes instability[1]. The elastic structural 
analysis of the second-order effect can be performed in the finite element method by adding the geometric stiffness matrix to the elastic linear matrix "mechanical stiffness" for the beam column element. The geometric stiffness, as shown in (1), is not a function of the mechanical properties of the element and is only a function of the element's length and the force in the element. Hence, the term "geometric" stiffness matrix is introduced so that the matrix has a different name from the "mechanical" stiffness matrix, which is based on the physical properties of the element. The geometric stiffness exists in all structures; however, it becomes important only if it is large compared to the mechanical stiffness of the structural system [2]. To use this stiffness, the element must be divided into small segments between the load points for more accuracy. Several trials must be done when using the geometric stiffness till achieving the equilibrium, as shown in equation (3).

$$
\begin{aligned}
& {\left[\begin{array}{c}
F_{i} \\
M_{i} \\
F_{j} \\
M_{j}
\end{array}\right] }=\frac{T}{30 L}\left[\begin{array}{cccc}
36 & 3 L & -36 & 3 L \\
3 L & 4 L^{2} & -3 L & -L^{2} \\
-36 & -3 L & 36 & -3 L \\
3 L & -L^{2} & -3 L & 4 L^{2}
\end{array}\right]\left[\begin{array}{c}
v_{i} \\
\theta_{i} \\
v_{j} \\
\theta_{j}
\end{array}\right] \\
& \text { or } F_{G}=K_{G} v, \\
& {\left[\begin{array}{c}
F_{i} \\
M_{i} \\
F_{j} \\
M_{j}
\end{array}\right]=\frac{E I}{L^{3}}\left[\begin{array}{cccc}
12 & 6 L & -12 & 6 L \\
6 L & 4 L^{2} & -6 L & -2 L^{2} \\
-12 & -6 L & 12 & -6 L \\
-6 L & -2 L^{2} & -6 L & 4 L^{2}
\end{array}\right]\left[\begin{array}{c}
v_{i} \\
\theta_{i} \\
v_{j} \\
\theta_{j}
\end{array}\right] } \\
& \text { or } F_{E}=K_{E} v, \\
& F_{T}=F_{E}+F_{G} \\
&= \\
&=
\end{aligned}
$$

A nonlinear second-order frame analysis procedure can be performed to analyze reinforced concrete columns that are a part of frames. In order to account for second-order effects due to geometric and material nonlinearities, the theoretical model (computer software) uses classical stiffness analysis of linear elastic two-dimensional structural frames, the iterative technique combined with an incremental method for computing load-deflection behavior and failure load of the frame, frame discretization to account for column chord $(P-\Delta)$ effects and axial load-bending moment-curvature $(P-M-\phi)$ relationships to account for effects of nonlinear material behavior [3].

\section{Computing of the Additional Moments in Different Codes}

Design of RC long column must consider the induced additional moments in these columns due to the axial load and occurred lateral deflection. Computing the additional moments for design requires simplified procedure and adequate accuracy. There are many methods that have been derived from modifying the results of a first-order analysis to approximate the second-order effects as which are recommended in a lot of codes such as American code ACI [4] and Canadian code CSA [5]. These codes permit the use of a moment magnifier approach to approximate the second-order moments due to the axial load acting through the lateral deflection caused by the end moments acting on a column. In the moment-magnifier analysis, unequal end moments are applied on the column shown in Figure 1(a). The column is replaced with a similar column subjected to equal moments at both ends, which is shown in Figure 1(b). The bending moments are chosen where the maximum magnified moment is the same in both columns. The expression for the factor $C_{m}$ was originally derived for use in the steel beam-columns design and was adopted without change for concrete design.

$M_{2}, M_{1}$ are the larger and smaller end moments of the first-order analysis, respectively. If a single curvature bending occurred by the moments $M_{1}$ and $M_{2}, M_{1} / M_{2}$ is positive. However, if the moments cause double curvature, $M_{1} / M_{2}$ is negative. The moment magnifier equation in the cases of no sway according to ACI,

$$
\begin{aligned}
M_{c} & =\delta_{n s} \cdot M_{2} \\
& =C_{m} \delta_{1} M_{2} \geq M_{2} . \\
C_{m} & =0.6+0.4 \frac{M_{1}}{M_{2}},
\end{aligned}
$$

Chen and Lui [6] explain that $C_{m}$ and $\delta_{1}$ for pin-ended columns subjected to end moments can be derived from the basic differential equation governing the elastic in-plane behavior of a column. ACI Code goes on to define $\delta_{n s}$ as follows:

$$
\delta_{n s}=\frac{C_{m}}{1-0.75 P / P_{c}} .
$$

The 0.75 factor in equation (6) is the stiffness reduction factor $\phi K$, which is based on the probability of under strength of a single isolated slender column.

In Eurocode [7], three methods applied for second-order impacts analysis are pointed:

Simplified method: based on nominal stiffness (MNS), simplified method: based on nominal curvature (MNC), and overall method: based on nonlinear second-order analysis.

The method of nominal stiffness is based on the critical force due to the buckling computed for the nominal stiffness of the analyzed member. It is recommended that the material nonlinearity, creep, and cracking, which have an effect on the conduct of the structure members, are taken into consideration. The design moment in the members subjected to the bending moment and an axial force which includes the impact of the first and second-order effects can be illustrated as a bending moment boosted by the factor described below: 


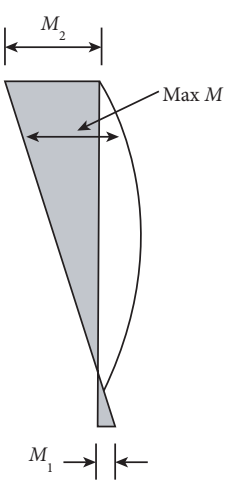

(a)

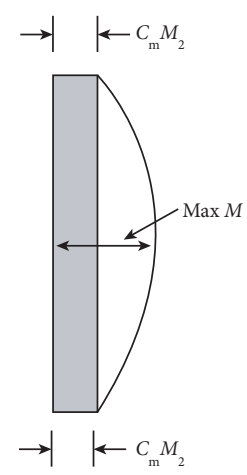

(b)

Figure 1: Equivalent moment factor $C_{m}$. (a) Actual moments at failure. (b) Equivalent moments at failure.

$$
\begin{aligned}
M_{E d} & =M_{0 E d}+M_{2} \\
& =M_{0 E d}+M_{0 E d} \cdot \frac{\beta}{\left(N_{B} / N_{E d}\right)-1} \\
& =M_{0 E d} \cdot\left[1+\frac{\beta}{\left(N_{B} / N_{E d}\right)-1}\right],
\end{aligned}
$$

where $M_{O E d}$ is the $1^{\text {st }}$ order moment, including the effect of imperfections, $M_{2}$ is the nominal $2^{\text {nd }}$ order moment, $N_{B}$ is the buckling load based on nominal stiffness, $N_{E d}$ is the design value of the axial load, $\beta$ is the factor which depends on the distribution of the $1^{\text {st }}$ and $2^{\text {nd }}$ order moments.

The method of nominal curvature allows for the calculation of the second-order moment based on the assumed curvature distribution (which responds to the first-order moment increased by the second-order effects) on the length of the member. The distribution of the total curvature can be either parabolic or sinusoidal.

The value of the II order moment can be calculated as follows:

$$
M_{2}=N_{E d} \cdot e_{2},
$$

where $N_{E d}$ is the design value of the axial load, $e_{2}$ is the deflection calculated by taking into account such parameters as creep, the intensity of reinforced and also distribution of the reinforcement over the height of the cross-section

$$
e_{2}=\frac{1}{r} \cdot \frac{l_{0}^{2}}{c},
$$

where $c$ is the factor depending on the curvature distribution, $l_{0}$ is the effective length, and $1 / r$ is the curvature.

According to Egyptian Code [8], $\left(M_{\text {add }}\right)$ is induced by the deflection $(\delta)$ given by the following:

$$
M_{\text {add }}=P . \delta .
$$

If the column is long in $t$ direction,

$$
\begin{aligned}
\delta_{t} & =\frac{\lambda_{t}{ }^{2} t}{2000}, \\
M_{\text {add }} & =P . \delta_{t} .
\end{aligned}
$$

However, if the column is long in $b$ direction,

$$
\begin{aligned}
\delta_{b} & =\frac{\lambda_{b}^{2} b}{2000}, \\
M_{\text {add }} & =P \cdot \delta_{b}, \\
\lambda_{b} & =\frac{H_{e}}{b}, \\
H_{e} & =k \cdot H_{0},
\end{aligned}
$$

where $H_{e}$ is the effective height of the column, $H_{0}$ is clear height of the column, $k$ is length factor which depends on the conditions of the end column and the bracing conditions.

The presented equations in the mentioned codes depend on their derivation on the isolated analysis for the long column and computed maximum bending moments induced through the height of the column. The additional moments analysis at the joints between the column and connected beams did not receive any interest in the different codes. Only the recommended equations in these codes take into account the effect of the connected beams on the additional moments through the column height by dealing with the effective length of the column, not the total length. In this research, a new moment magnifiers matrix will be presented in a derived equation for an equivalent column to compute the additional moments of the braced long column, including the moments at the joints between the column and the connected beams. In this model, the additional moment diagram of a braced long column and its deformations can be computed taking into consideration the second-order effect of the axial load and the inverse moments at the connection between the columns and the beams. Material nonlinearities will be considered by modifying the elastic flexural rigidity (EI) to effective flexural stiffness computed according to ACI (2019). 


\section{Lateral Displacements in a Long Column under End Moments}

In the first-order analysis, the curvature equation for a long column under equal end moments as shown in Figure 2 can be expressed as follows:

$$
\frac{\mathrm{d}^{2} y}{\mathrm{~d} x^{2}}=-\frac{M}{\mathrm{EI}},
$$

where $M=M_{0}$

$$
\begin{aligned}
& \frac{\mathrm{d} y}{\mathrm{~d} x}=-\frac{M_{0}}{E I} x+C_{1}, \\
& \delta_{0}=-\frac{1}{E I}\left[\frac{M_{0} x^{2}}{2}\right]+C_{1} x+C_{2} .
\end{aligned}
$$

By applying the boundary conditions, it is found that $C_{2}=0$ and $C_{1}=M_{0} L / 2 \mathrm{EI}$.

And equation (15) becomes as follows:

$$
\delta_{0}=\frac{1}{\mathrm{EI}}\left[-\frac{M_{0} x^{2}}{2}+\left(\frac{M_{0} L}{2}\right) x\right] .
$$

Maximum lateral displacement at the mid-span of the column can be expressed as follows:

$$
\left(\delta_{0}\right)_{\max }=\frac{M_{0} L^{2}}{8 \mathrm{EI}} .
$$

Due to the second-order effect, the lateral displacement of the column increases and it can be expressed as $\left(\delta_{o}+\delta_{a}\right)$, where $\delta_{a}$ is the lateral displacement which is caused by the additional moments. To compute the maximum additional lateral displacement at the middle span of the column, the virtual work method can be used. As observed that the deformation shape of the first-order analysis is $2^{\text {nd }}$ curve as shown in equation (16). As a result, additional displacement and the additional moment diagram will be $2^{\text {nd }}$ curve, where $M_{\text {add }}=P .\left(\delta_{0}+\delta_{a}\right)$.

The additional lateral displacement can be found as follows:

$$
\begin{aligned}
\delta_{a} & =\int_{0}^{L} M_{\mathrm{add}} M_{11} \mathrm{~d} x, \\
M_{\mathrm{add}} & =P \cdot\left(\delta_{0}+\delta_{a}\right), \\
\delta_{a} & =\frac{1}{\mathrm{EI}}\left(2 * \frac{2}{3} P\left(\delta_{0}+\delta_{a}\right) \cdot \frac{L}{2} \cdot \frac{5}{8} \cdot \frac{L}{4}\right), \\
\delta_{a} & =\frac{\delta_{0} 5 P L^{2} / 48 \mathrm{EI}}{1-5 P L^{2} / 48 \mathrm{EI}}, \\
\text { But } \frac{1}{P_{e}} & =\frac{5 L^{2}}{48 \mathrm{EI}} \text { where } P_{e} \text { is Euler load, } \\
\delta_{a} & =\frac{\delta_{0} P / P_{e}}{1-P / P_{e}} .
\end{aligned}
$$
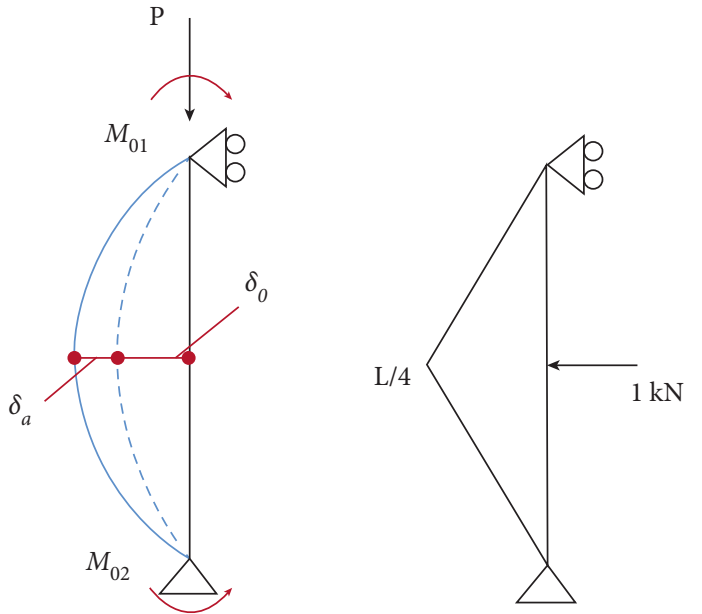

Figure 2: Deformed shape of a long column under end moments.

The final maximum displacement will be as follows:

$$
\begin{array}{r}
\delta_{f}=\frac{\delta_{0} P / P_{e}}{1-P / P_{e}}+\delta_{0}, \\
\text { It can be put } \eta=\frac{1}{1-P / P_{e}}, \\
\text { So } \delta_{f}=\delta_{0} \eta .
\end{array}
$$
follows:

The maximum additional moments can be formulated as

$$
M_{\mathrm{add}}=P \delta_{o} \eta
$$

When the column is deformed under unequal end moments, the bigger moment can be divided into two parts $M=M_{0}+\Delta M$, and the column will be considered under equal end moments $\left(M_{0}\right)$ which was illustrated previously and one end moment $(\Delta M)$.

In the first-order analysis, the curvature equation for a long column under one end moment can be expressed as follows:

$$
\begin{gathered}
\frac{\mathrm{d}^{2} y}{\mathrm{~d} x^{2}}=-\frac{M}{E I}, \\
M=R x \text { where } \mathrm{R} \text { is the reaction and equals } R=\frac{\Delta M}{L}, \\
\frac{\mathrm{d} y}{\mathrm{~d} x}=-\frac{1}{E I}\left(\frac{R x^{2}}{2 E I}\right)+C_{1}, \\
\delta_{\Delta 0}=-\frac{1}{\mathrm{EI}}\left[\frac{R x^{3}}{6}\right]+C_{1} x+C_{2},
\end{gathered}
$$

where $\delta_{\Delta 0}$ : the lateral displacement due to $\Delta M$.

By applying the boundary conditions, $C_{2}=0$, $C_{1}=R L^{2} / 6 \mathrm{EI}$ and put $R=\Delta M / L$ 


$$
\delta_{\Delta 0}=\frac{1}{\mathrm{EI}}\left[-\frac{\Delta M x^{3}}{6 L}+\frac{\Delta M L x}{6}\right] .
$$

As shown in equation (24), the deflection curve due to $\Delta M$ is 3 rd-degree parabolic curve, and the maximum deflection occurs when $\mathrm{d} \delta / \mathrm{d} x=0$

$$
\text { Thus } \frac{\mathrm{d} y}{\mathrm{~d} x}=\frac{1}{\mathrm{EI}}\left(\frac{M x^{2}}{2 L}+\frac{\Delta M L}{6}\right)=0 \text {. }
$$

By solving equation (26), max. lateral displacement due to $\Delta M$ will be at. $x=L / \sqrt{3}$ and Max. lateral displacement due to $\Delta M$ is given by the following:

$$
\delta_{\Delta 0}=\frac{\Delta M L^{2}}{9 \sqrt{3} \mathrm{EI}}
$$

Also, due to the second-order effect, the lateral displacement of the column increases, and it can be expressed as $\left(\delta_{\Delta o}+\delta_{\Delta a}\right)$, where $\delta_{a \Delta}$ is the lateral displacement which is caused by the additional moments. Considering that the deformation shape of the first-order analysis is $3^{\text {rd }}$ curve as shown in equation (25), also additional displacement and the additional moment diagram will be $3^{\text {rd }}$ curve. Similarly, the maximum lateral displacement due to $\Delta M$ in second-order analysis can be found as the same manner of the case of equal end moments as in the following equation:

$$
\begin{array}{r}
\delta_{f \Delta}=\frac{\delta_{\Delta 0}}{1-P / P_{e}} \\
=\delta_{\Delta_{0}} \eta .
\end{array}
$$

The additional moments can be formulated as follows:

$$
M_{\mathrm{add}}=P \delta_{\Delta o} \eta \text {. }
$$

\section{Equivalent Lateral Load for the Second-Order Effect in a Long Braced Column}

As shown in section (2), when the column is loaded with an equal end moment, the deformed shape of the column in the second-order analysis was $2^{\text {nd }}$-degree curve. As a result, the expected additional bending moment diagram will be as the induced bending moment from the regular distributed load. Thus, the long column in the second-order effect can be replaced in a beam element subjected to an equivalent regular distributed load, and the equivalent load can be computed as follows:

$$
\frac{w_{e q R} L^{2}}{8}=P \eta\left(\frac{M_{0} L^{2}}{8 E I}\right),
$$

where $w_{e q R} L^{2} / 8$ : max. moment due to equivalent regular load, $P \eta\left(M_{0} L^{2} / 8 \mathrm{EI}\right)$ : max. moment due to second-order analysis, $w_{\text {eqR }}$ : equivalent regular distributed load.

$$
w_{e q R}=P \eta \frac{M_{0}}{\mathrm{EI}} \text {. }
$$

In similar to the column under equally end moment, the deformed shape in the second-order analysis due to one end moment as shown in Figure 3 is 3rd-degree curve and the additional bending moment diagram in the second-order analysis will be as the induced bending moment from the triangular distributed load. Also, in this case, the long column in the second-order effect can be replaced in a beam that is subjected to equivalent triangular distributed load, and the equivalent load can be computed as follows:

$$
\frac{w_{e q \Delta} L^{2}}{9 \sqrt{3}}=P \eta\left(\frac{\Delta M L^{2}}{9 \sqrt{3} \mathrm{EI}}\right),
$$

where $w_{e q \Delta} L^{2} / 9 \sqrt{3}$ is the max. moment due to equivalent triangular load, $P \eta\left(\Delta M L^{2} / 9 \sqrt{3} \mathrm{EI}\right)$ is the max. moment due to second-order analysis, and $w_{e q \Delta}$ is the equivalent triangular distributed load

$$
w_{e q \Delta}=P \eta \frac{\Delta M}{\mathrm{EI}}
$$

\section{New Moment Magnifiers Matrix of Braced Long Columns}

5.1. Equivalent Column Modeling. Based on the equivalent column concept, Afefy and El-Tony [9] have shown equivalent pin-ended columns for columns bent in either single or double curvature modes where the impact of end eccentricity ratio was related to the equivalent column length. They deduced that the equivalent column concept can be generalized to simplify columns bent in single curvature modes with different end eccentricities combinations to pin-ended axially loaded columns. Furthermore, the equivalent column concept can be carried out for a specific state of a column bent in double curvature mode.

Here, in the suggested equivalent column model, the column at any structure will be analyzed as an isolated element. The equivalent column was represented as an element restricted by a rotational spring support at its ends and it is subjected to lateral distributed loads. The lateral distributed loads have the same influence of the secondorder effect on the induced additional moments in the long column. Column (1), for example, in the shown closed frame in Figure 4 will be analyzed to illustrate the model. The column will be modeled as a pin-supported member restricted by the connected beams which are as rotational spring supports. Computing the rotational stiffness $\left(K_{\theta}\right)$ of these beams will be discussed later. The second-order analysis of the modeled column in Figure 5 can be divided into two parts.

The first part is concerned with the deformation due to the end moments of the first-order analysis without the existence of the reaction moments of the rotational spring. The induced moments of the second-order effect is equivalent to the induced moments of trapezoidal load. Thus, the column can be represented as a pin-supported column subjected to triangular distributed loads $w_{e q 1}$ and $w_{e q 2}$ 

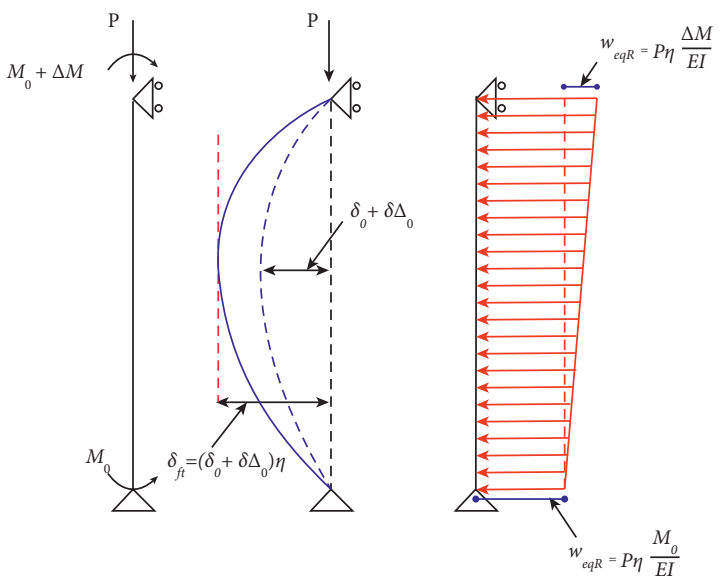

Figure 3: Equivalent lateral load for the second-order effect of a long column.

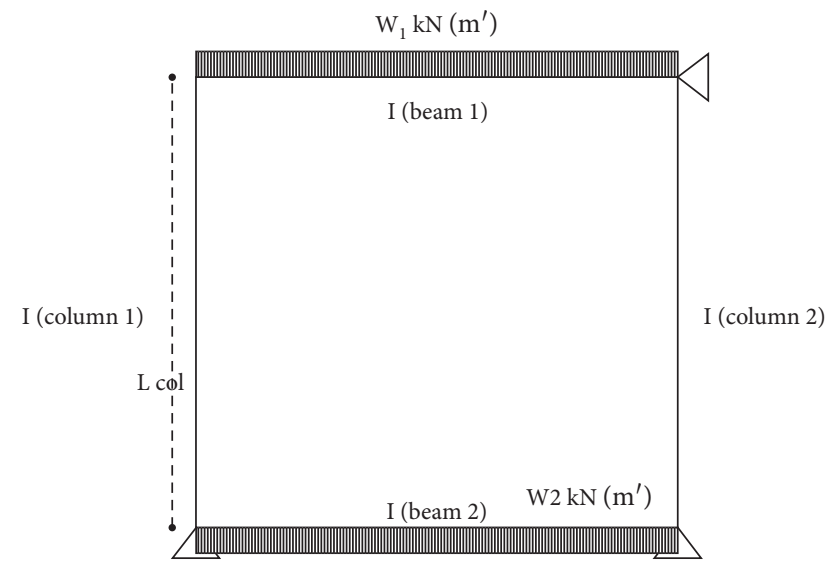

$\mathrm{Lb}$

Figure 4: Closed frame as an example.

$$
w_{e q 1}=p \eta \frac{M_{01}}{\mathrm{EI}}
$$

where $w_{e q 1}$ is the triangular equivalent load for the second effect due to moment at the column end at the beam of higher stiffness and $M_{01}$ is the the end moment at the beam of higher stiffness due to first order.

$$
w_{\text {eq } 2}=p \eta \frac{M_{02}}{\mathrm{EI}} .
$$

$w_{e q 2}$ is the triangular equivalent load for the second effect due to the moment at the column end at the beam of lower stiffness.

$M_{02}$ is the the end moment at the beam of lower stiffness due to first order.

The second part is concerned with the deformation due to the reaction moments of the spring rotational support only. Also, the column in the second effect will be represented as a pin-supported column subjected to triangular distributed loads $w_{e q_{1}^{*}}$ and. $w_{e q_{2}^{*}}$

$w_{e q_{1}^{*}}$ is the triangular equivalent load for the second effect due to the reaction moment of spring rotational support at the beam of higher stiffness, $M_{1}$ : the additional moment of spring rotational support at the beam of higher stiffness.

$$
w_{e q_{1}^{*}}=p \eta \frac{M_{1}}{\mathrm{EI}}
$$

$w_{e q_{2}^{*}}$ is the triangular equivalent load for the second effect due to the reaction moment of spring rotational support at the beam of lower stiffness, $M_{2}$ : the additional moment of spring rotational support at the beam of lower stiffness.

$$
w_{e q_{2}^{*}}=p \eta \frac{M_{2}}{\mathrm{EI}} .
$$

By arranging the linear stiffness matrix of a beam element for computing the moments of the modeled column in Figure 5, the formula will be as follows:

$$
\frac{\mathrm{EI}}{L^{3}}\left[\begin{array}{cc}
4 L^{2} & 2 L^{2} \\
2 L^{2} & 4 L^{2}
\end{array}\right]\left[\begin{array}{l}
\theta_{1} \\
\theta_{2}
\end{array}\right]+\left[\begin{array}{l}
\frac{-w_{e q 1} L^{2}}{20}-\frac{w_{e q 2} L^{2}}{30}+\frac{P \eta M_{2} L^{2}}{30 E I}-\frac{P \eta M_{1} L^{2}}{20 E I} \\
\frac{w_{e q 1} L^{2}}{30}+\frac{w_{e q 2} L^{2}}{20}-\frac{P \eta M_{2} L^{2}}{20 E I}+\frac{P \eta M_{1} L^{2}}{30 E I}
\end{array}\right]=\left[\begin{array}{l}
M_{1} \\
M_{2}
\end{array}\right] .
$$

The terms in equation (37) are as shown below:$$
K_{0}=\frac{\mathrm{EI}}{L^{3}},
$$$$
C=\frac{P \eta L^{2}}{\mathrm{EI}},
$$

$$
\begin{gathered}
\theta_{1}=\frac{-M_{1}}{K_{\theta 1}}, \\
\theta_{2}=\frac{-M_{2}}{K_{\theta 2}} .
\end{gathered}
$$

The matrix in (37) can be divided into the following: 


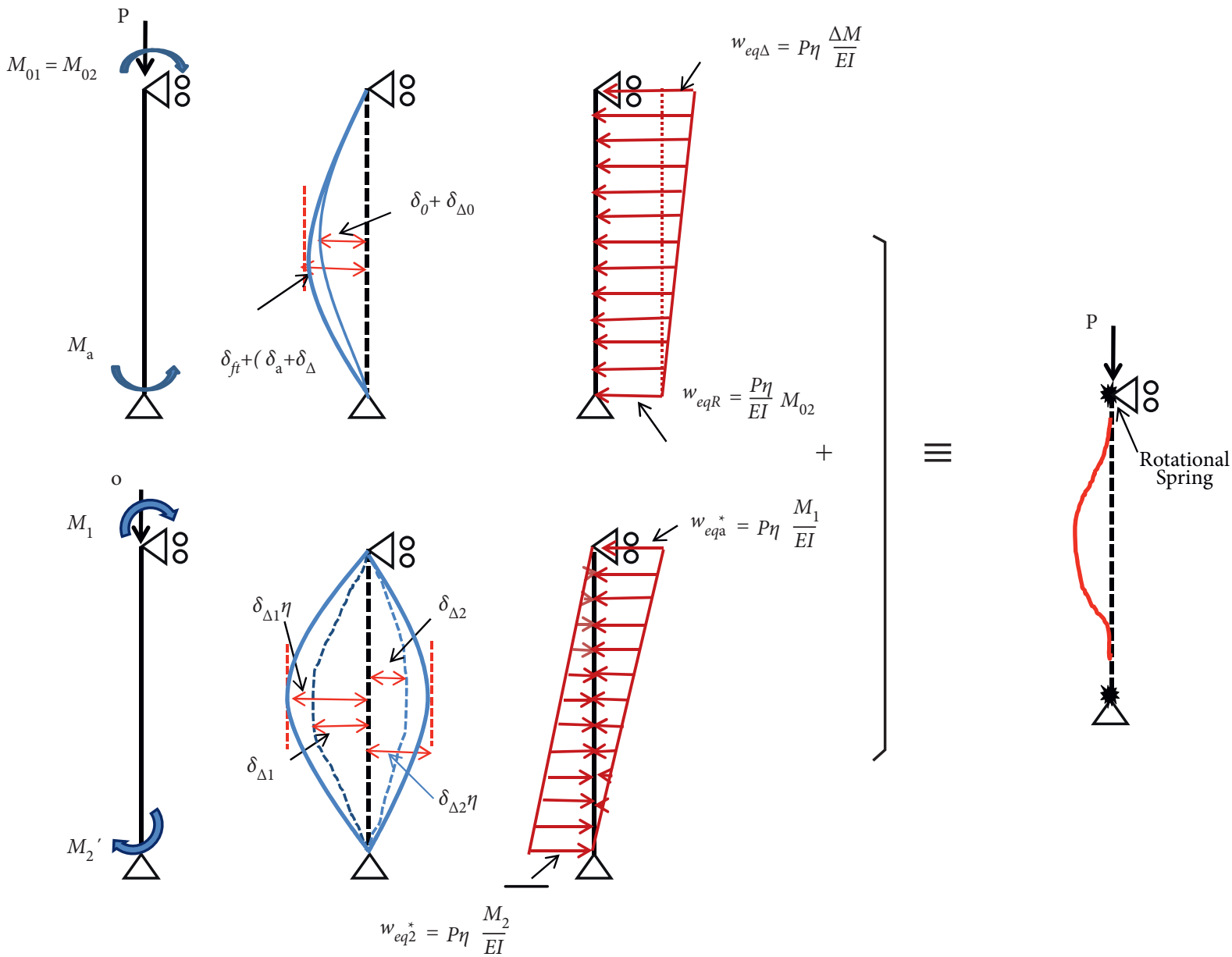

FIGURE 5: Equivalent column modeling for the restricted column (1) in the closed frame.

$$
\begin{gathered}
{\left[-1-\frac{4 L^{2} K_{0}}{K_{\theta 1}}+\frac{C}{20}\right] M_{1}+\left[-\frac{2 L^{2} K_{0}}{K_{\theta 2}}+\frac{C}{30}\right] M_{2}=\frac{w_{e q 1} L^{2}}{20}+\frac{w_{e q 2} L^{2}}{30}} \\
{\left[-\frac{2 L^{2} K_{0}}{K_{\theta 1}}+\frac{C}{30}\right] M_{1}+\left[-1-\frac{4 L^{2} K_{0}}{K_{\theta 2}}-\frac{C}{20}\right] M_{2}=\frac{-w_{e q 1} L^{2}}{30}-\frac{w_{e q 2} L^{2}}{20} .}
\end{gathered}
$$

The final formula to compute the additional moments at the column ends will be as follows:

$$
\left[\begin{array}{l}
M_{1} \\
M_{2}
\end{array}\right]=C\left[\begin{array}{cc}
-1-\frac{4 L^{2} K_{0}}{K_{\theta 1}}-\frac{C}{20} & -\frac{2 L^{2} K_{0}}{K_{\theta 2}}+\frac{C}{30} \\
-\frac{2 L^{2} K_{0}}{K_{\theta 1}}+\frac{C}{30} & -1-\frac{4 L^{2} K_{0}}{K_{\theta 2}}-\frac{C}{20}
\end{array}\right]^{-1}\left[\begin{array}{c}
\frac{M_{01}}{20}+\frac{M_{02}}{30} \\
-\frac{M_{01}}{30}-\frac{M_{02}}{20}
\end{array}\right] .
$$

Equation (40) can be rewritten as follows:

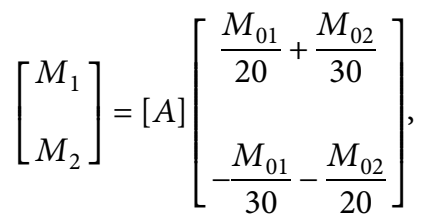

where $[A]$ is considered as moment magnifiers matrix for the end additional moments and it is equal to the following:

$$
[A]=C\left[\begin{array}{cc}
-1-\frac{4 L^{2} K_{0}}{K_{\theta 1}}-\frac{C}{20} & -\frac{2 L^{2} K_{0}}{K_{\theta 2}}+\frac{C}{30} \\
-\frac{2 L^{2} K_{0}}{K_{\theta 1}}+\frac{C}{30} & -1-\frac{4 L^{2} K_{0}}{K_{\theta 2}}-\frac{C}{20}
\end{array}\right]^{-1}
$$

Just the additional moments at the column ends were computed, the final load of the equivalent column will be as shown in Figure 6. 


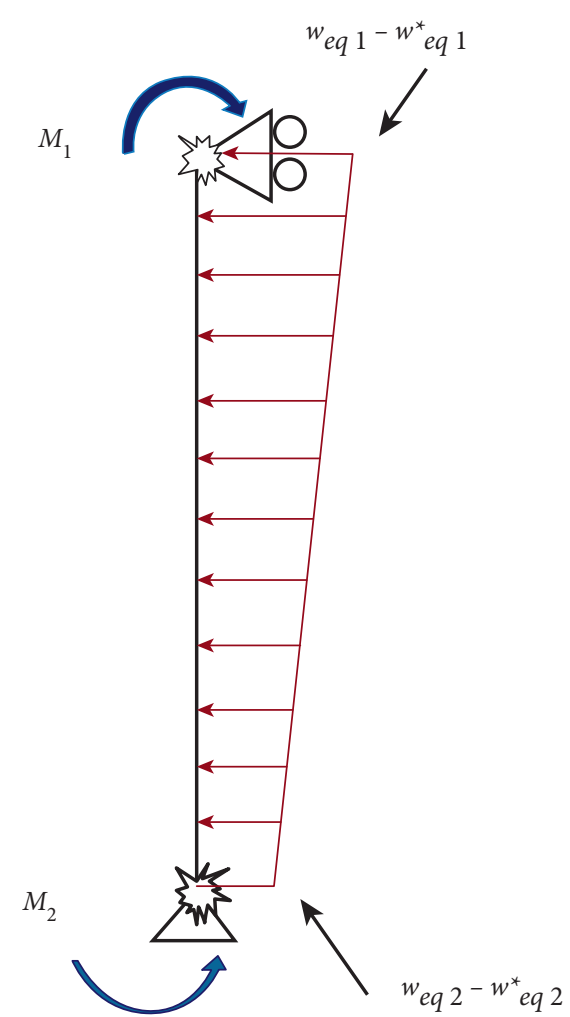

FIgURE 6: Final load of the equivalent column.

Through the model in Figure 6, the additional bending moment at any section can be computed and an additional bending moment diagram can be formed. Also, by using one of the methods of structural analysis, such as the virtual work method or area moment method, the additional lateral displacement and rotations at any point can be calculated. The total lateral displacement also can be computed easily, by dividing the additional bending moment at any section by the axial load $\left(\delta_{\text {final }}=M_{\text {add. }} / P\right)$.

\section{Approximate Rotational Stiffnesses for the Column at Upper and Lower Joints}

The connected beams which represent the rotational stiffness of the upper and lower joints of the column $\left(K_{b}\right)$ can be approximately computed by applying one unit of the moment toward the end of the connected beams with the studied column as shown in Figure 7. The opposite end of the beam is considered as rotational restricted end by another pin column (adjust column to the studied column). Rotation of the beam end $\left(\theta_{b}\right)$ under the unit moment can be calculated. Then, the rotational stiffness will be computed as $K_{b}=1 / \theta_{b}$.

The rotation at the loaded end of the adjacent column is computed as follows:

$$
\begin{array}{r}
\theta_{\text {col. }}=\frac{1}{\mathrm{EI}_{. c o l}} \int_{0}^{L} M_{o} \cdot M_{1} \\
=\frac{L_{\text {.Acol. }}}{3 \mathrm{EI}_{\text {.Acol }}},
\end{array}
$$

where $L_{A c o l}$ and $I_{A c o l}$ are the length and moment of inertia of the adjacent column.

The rotational stiffness of the adjacent column to the beam is as follows:

$$
K_{\text {Acol }}=\frac{3 \mathrm{EI}_{. c o l}}{L_{. c o l}} .
$$

Due to the unit moment at the beam end, the reaction moment $\mathrm{M} *$ at the opposite end of the beam can be found by the force method, as follows:

$$
M^{*}=\left(\frac{L_{b} / 6}{\mathrm{EI}_{b} / K_{A . c o l}+L_{b} / 3}\right) .
$$

By using the virtual work, the rotation at the loaded end of the beam can be determined as follows:

$$
\theta_{b}=\frac{1}{E I_{b}}\left[\frac{1}{2} \cdot \frac{L_{b}}{3} \cdot 2-\frac{L_{b}^{2}}{12} \cdot \frac{1}{3} \frac{1}{\mathrm{EI}_{b} / K_{A c o l}+L_{b} / 3}+\frac{L_{b}^{2}}{36} \cdot \frac{1}{3}\left[\frac{1}{\mathrm{EI}_{b} / K_{A c o l}+L_{b} / 3}\right]^{2}\right]
$$

The rotational rigidity of the connected beam end for the studied column can be expressed as follows:

$$
K_{b}=\frac{\mathrm{EI}_{b}}{L_{b}\left((1 / 3)-\left(L_{b} / 36\right) \cdot\left(1 / \mathrm{EI}_{b} / K_{A c o l}+L_{b} / 3\right)+L_{b}^{2} / 108\left(1 / \mathrm{EI}_{b} / K_{A c o l}+L_{b} / 3\right)^{2}\right)},
$$

where $L_{b}$ and $E I_{b}$ are the length and moment of inertia of the connected beam.

In fact, most of the long columns are connected with beams that have stiffness bigger than or close to the column stiffness. Thus, the effect of the adjacent column, which as rotational spring for the beams, will be slight and (47) can be simplified as follows:

$$
K_{b}=\frac{3 \mathrm{EI}_{b}}{L_{b}}
$$




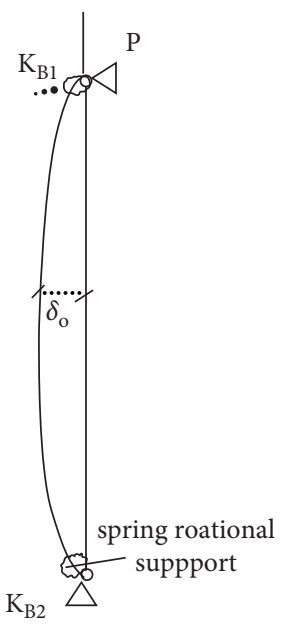

(a)
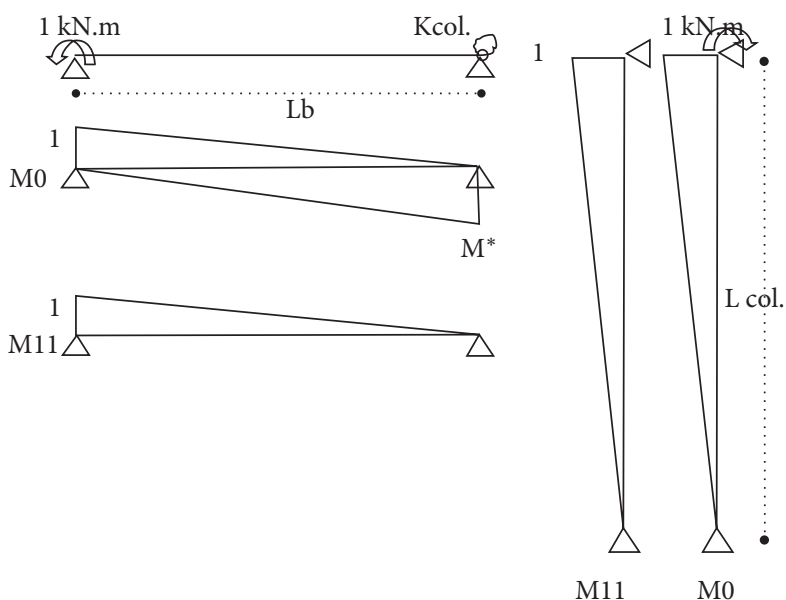

(c)

Figure 7: Rotational stiffness of the connected beam. (a) Studied column. (b) Connected beam. (c) Adjacent column.

\section{Computing the Additional Moments by Using the Equivalent Column with More Accuracy}

As mentioned before, the additional moments in a long column can be computed according to equation (41) as isolated column analysis. If there are other long columns adjacent to the studied columns, the additional moments of these columns will affect the additional moments of the studied column. For more accuracy, the effect of additional moments of adjacent columns must be considered, where a part of these moments will be transferred through the connected beams to the studied column. By one of the following two suggestions, the effect of the adjacent long columns can be taken into consideration.

7.1. Suggestion 1. Assume that the studied column is the left column in the shown closed frame in Figure 4 . In this suggestion, the additional moments in each column will be computed according to equation (41) as a separate analysis of each of them. Then the transferring ratio of the additional moments between the columns will be found. Each column will be considered as a rotational spring for both the bottom and top beams. The rotational stiffness of the columns will be computed in the same manner in section 5 , equation (44).

By using the force method, the transmitting moment from the right column to the left studied column at joint 1 as an example can be calculated as follows:

$$
\begin{aligned}
& M_{1}^{*}=\left(M_{\text {add }}\right)_{2} \cdot \frac{\left(L_{b} / 6 \mathrm{EI}_{b}\right)_{\mathrm{Top}}}{\left(L_{c o l} / 3 \mathrm{EI}_{c o l}+L_{b} / 3 \mathrm{EI}_{b \mathrm{Top}}\right)}, \\
& M_{1}^{*}=\left(M_{\mathrm{add}}\right)_{2} \cdot \alpha_{1},
\end{aligned}
$$

where $M_{1}^{*}$ is the transferred moment from the adjacent column (joint (2) to the studied column joint (1), $\alpha_{1}=\alpha_{2}$ : factor of transferring ratio by the top beam $=$ $\left(L_{b} / 6 \mathrm{EI}_{b}\right)_{\mathrm{Top}} /\left(L_{c o l} / 3 \mathrm{EI}_{c o l}+\left(L_{b} / 3 \mathrm{EI}_{b}\right)_{\mathrm{Top}}\right),\left(M_{a d d}\right)_{2}$ : the additional moment at joint 2 of the adjacent column.

$$
\begin{aligned}
& M_{3}^{*}=\left(M_{\text {add }}\right)_{4} \cdot \frac{\left(L_{b} / 6 \mathrm{EI}_{b}\right)_{\text {bottom }}}{\left(L_{c o l} / 3 \mathrm{EI}_{c o l}+\left(L_{b} / 3 \mathrm{EI}_{b}\right)_{\text {bottom }}\right)}, \\
& M_{3}^{*}=\left(M_{\text {add }}\right)_{4} \cdot \alpha_{2} .
\end{aligned}
$$

where $M_{3}^{*}$ is the the transferred moment from the adjacent column (joint 4) to the studied column joint (3), $\alpha_{3}=\alpha_{4}$ : factor of transferring ratio by the bottom beam $=$ $\left(L_{b} / 6 \mathrm{EI}_{b}\right)_{\text {bottom }} /\left(L_{c o l} / 3 \mathrm{EI}_{c o l}+\left(L_{b} / 3 \mathrm{EI}_{b}\right)_{\text {bottom }}\right)$

After obtaining the transmitting moment between the two columns, (41) can be carried out one time for the second-order effect of the transmitting moments. Also, this can be considered by modifying equation (41) as follows:

$$
\left[\begin{array}{l}
M_{1} \\
M_{3}
\end{array}\right]=[A]\left[\begin{array}{c}
\frac{\left(M_{01}+\alpha M_{\mathrm{add} 2}\right)}{20}+\frac{\left(M_{03}+\alpha M_{\mathrm{add} 4}\right)}{30} \\
-\frac{\left(M_{03}+\beta M_{\mathrm{add} 4}\right)}{30}-\frac{\left(M_{03}+\beta M_{\mathrm{add} 4}\right)}{20}
\end{array}\right]-\left[\begin{array}{l}
\alpha_{1} M_{\mathrm{add} 2} \\
\alpha_{3} M_{\mathrm{add} 4}
\end{array}\right] .
$$

Equation (51) takes into account the transmitting additional moments between two adjacent columns for one trial. The equation can be carried out for several trials till the ratio of transferred additional moment gets close to zero and it can be modified to include the effect of more adjacent columns. Whereas the deformations in reinforced concrete structures are small, thus the additional moments at the end of the long columns will not be large values. As a result, the expected transmitting moments will be small and it can be ignored, or one trial as maximum can be carried out. But for 


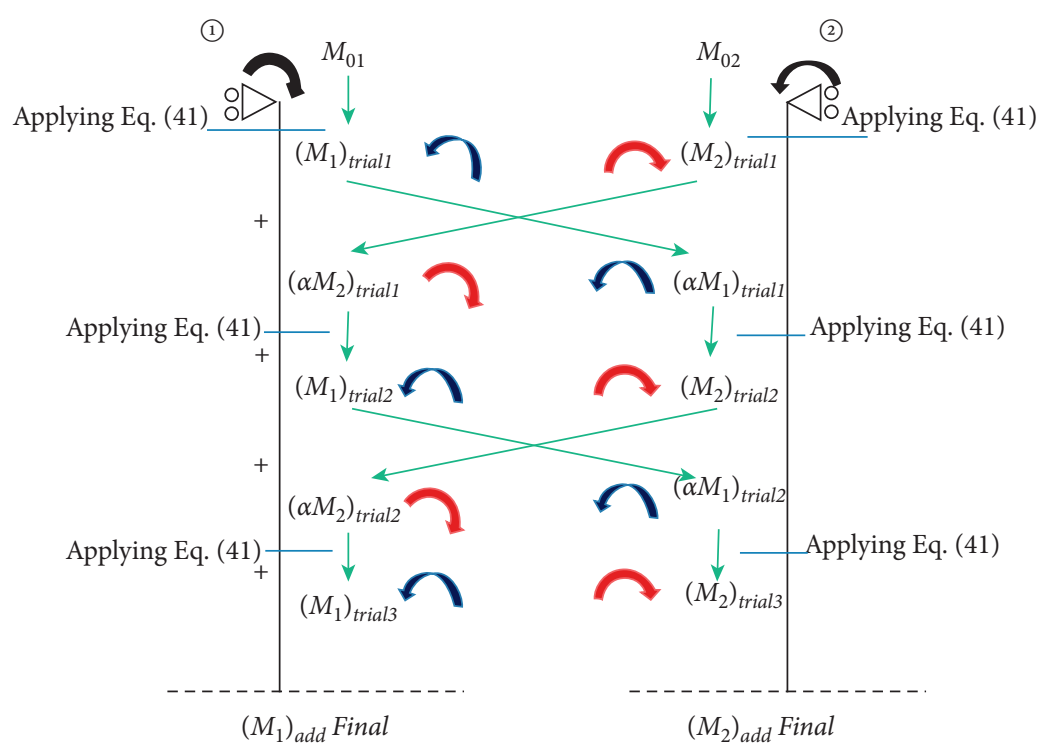

(a)

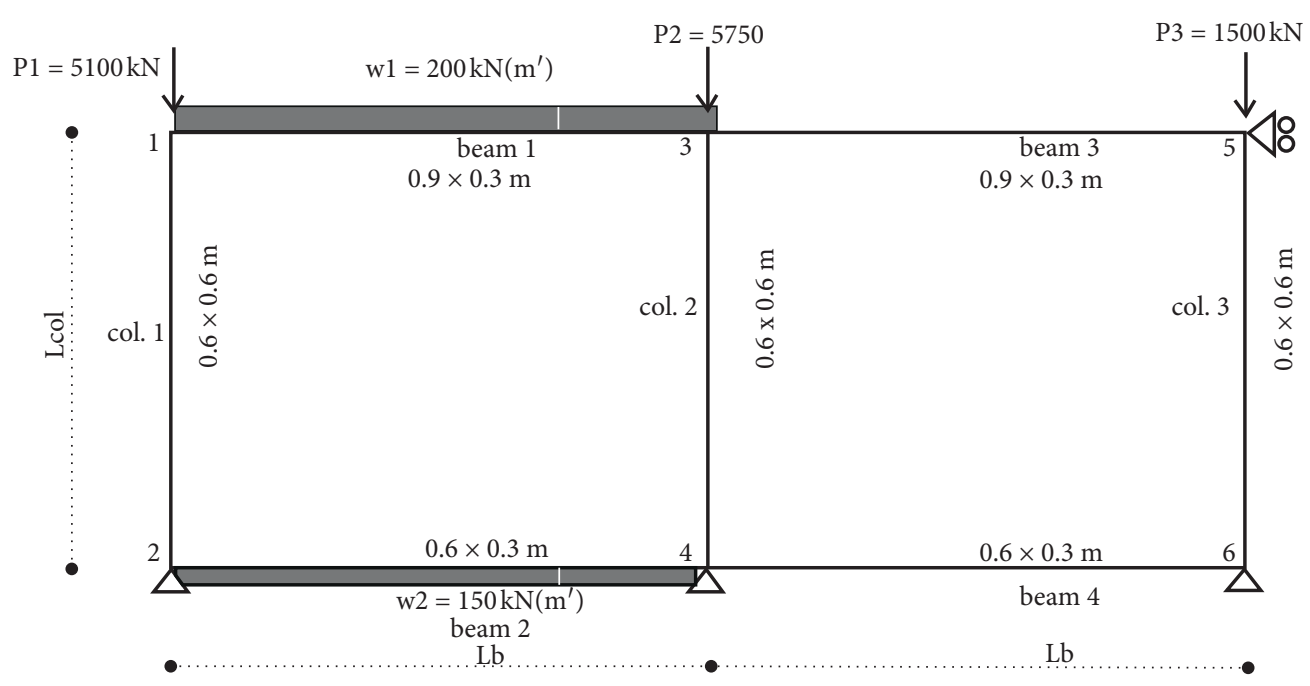

(b)

Figure 8: (a) Schematic method for the transmitting additional moments. (b) Multibays frame is an example of a structure that has more than two adjacent slender columns.

more accuracy, the effect of the additional moments of adjacent columns can be considered as in (51). The effect of adjacent additional moments can be considered schematic method as in Figures 8(a) and 9 presented the additional moments' transmission between the columns.

If a number of slender columns exist in the structure, as shown in Figure 8(b), equations (43) and (51) easily can be formulated as follows:
Equation (51) will become as follows:

$$
M_{\text {add }}=\sum_{\text {trial }=1}^{\text {trial }=n} M_{\text {triall }}+\alpha M_{\text {trial } 1}+M_{\text {trial } 2}+\alpha M_{\text {trial } 2}+\cdots \cdots+M_{\text {trial }(n)} \text {, }
$$

where $n$ is the trial number which at it the condition of $\left(\alpha M_{\text {trial(n) }} \cong\right.$ zero $)$ will be achieved 


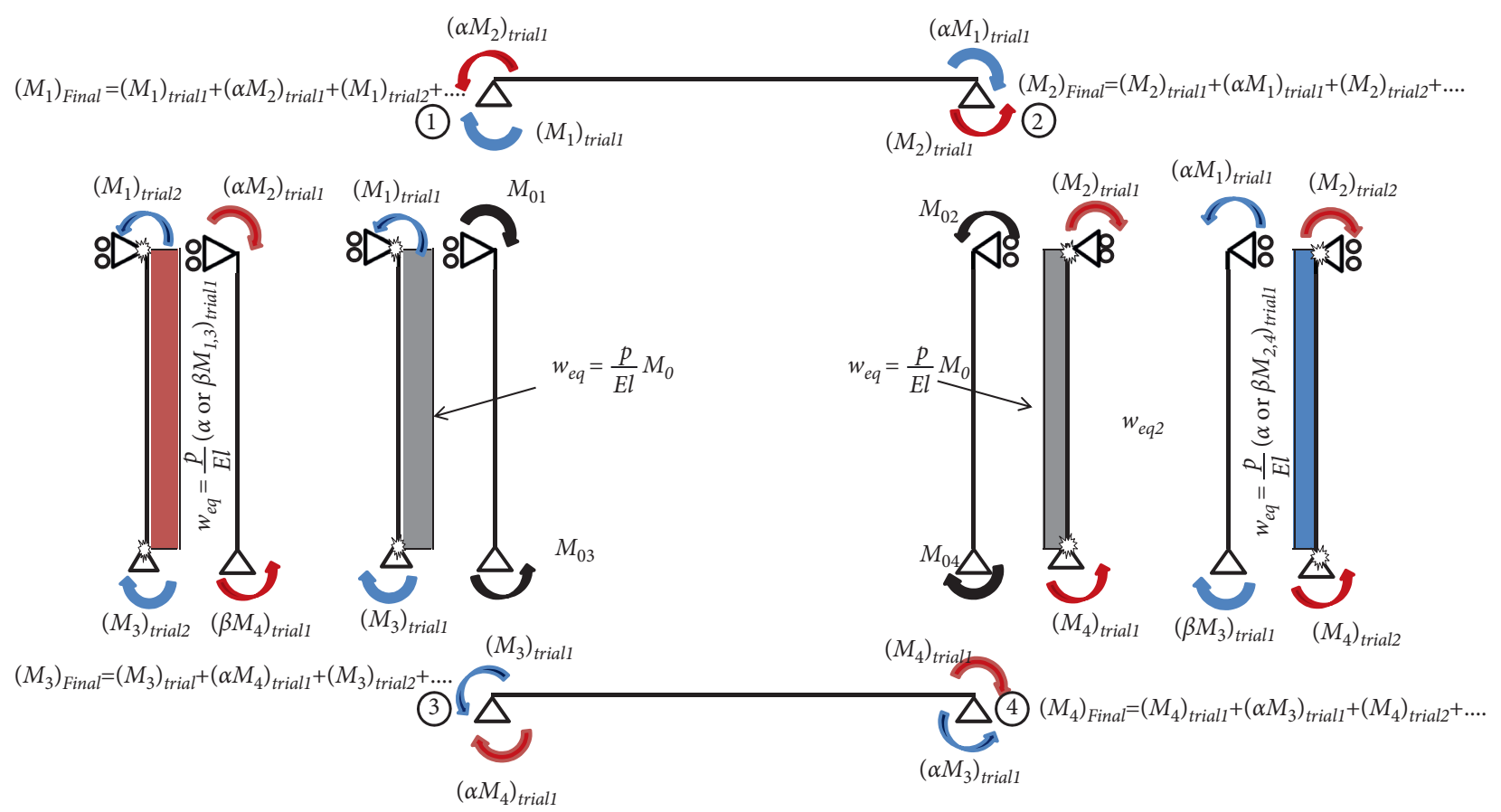

Figure 9: Additional moments transmission between the columns.

$$
\begin{aligned}
M_{\text {traill }} & =\left[\begin{array}{l}
M_{1} \\
M_{2} \\
M_{3} \\
M_{4} \\
M_{5} \\
M_{6}
\end{array}\right]_{\text {trail }} \\
& =\left[\begin{array}{cccccc}
\frac{1}{20} & \frac{1}{30} & 0 & 0 & 0 & 0 \\
-\frac{1}{30} & -\frac{1}{20} & 0 & 0 & 0 & 0 \\
0 & 0 & \frac{1}{20} & \frac{1}{30} & 0 & 0 \\
0 & 0 & -\frac{1}{30} & -\frac{1}{20} & 0 & 0 \\
0 & 0 & 0 & 0 & \frac{1}{20} & \frac{1}{30} \\
0 & 0 & 0 & 0 & -\frac{1}{30} & -\frac{1}{20}
\end{array}\right]\left[\begin{array}{l}
M_{01} \\
M_{02} \\
M_{03} \\
M_{04} \\
M_{05} \\
M_{06}
\end{array}\right],
\end{aligned}
$$




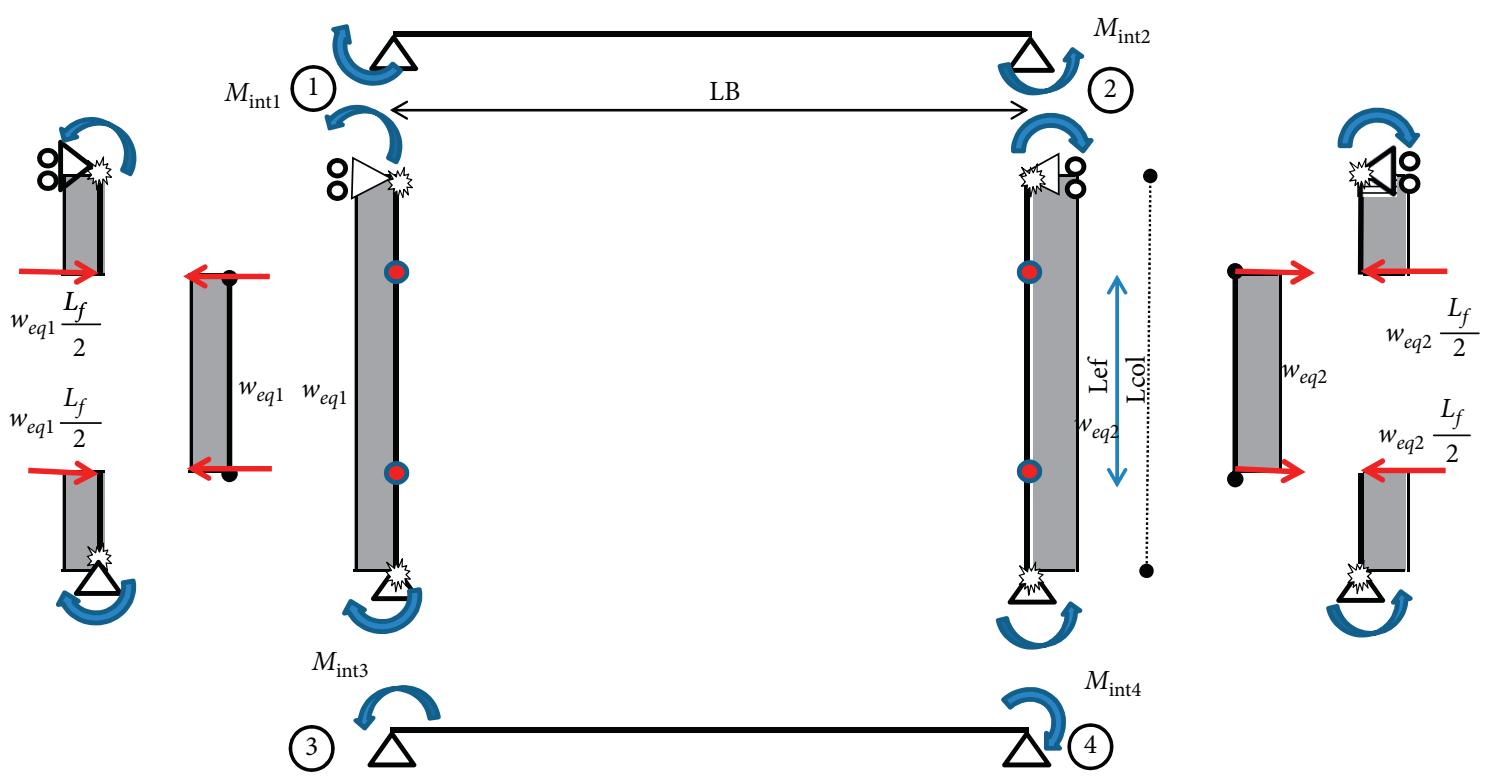

Figure 10: Approximate initial moments at the ends of the beam to calculate the relative rotational stiffness.

$$
\begin{aligned}
& M_{\text {transfferal }}=(\alpha) *[\mathrm{M}]_{\text {ttraill }} \\
& =\left[\begin{array}{c}
\alpha_{1} M_{3} \\
\alpha_{2} M_{4} \\
\alpha_{3 L} M_{1}+\alpha_{3 R} M_{5} \\
\alpha_{4 L} M_{1}+\alpha_{4 R} M_{6} \\
\alpha_{5} M_{3} \\
\alpha_{6} M_{4}
\end{array}\right], \\
& M_{\text {trail2 }}=\left[\begin{array}{c}
M_{1} \\
M_{2} \\
M_{3} \\
M_{4} \\
M_{5} \\
M_{6}
\end{array}\right]_{\text {trail2 }} \\
& {[A]\left[\begin{array}{cccccc}
\frac{1}{20} & \frac{1}{30} & 0 & 0 & 0 & 0 \\
-\frac{1}{30} & -\frac{1}{20} & 0 & 0 & 0 & 0 \\
0 & 0 & \frac{1}{20} & \frac{1}{30} & 0 & 0 \\
0 & 0 & -\frac{1}{30} & -\frac{1}{20} & 0 & 0 \\
0 & 0 & 0 & 0 & \frac{1}{20} & \frac{1}{30} \\
0 & 0 & 0 & 0 & -\frac{1}{30} & -\frac{1}{20}
\end{array}\right]\left[\begin{array}{c}
\alpha_{1} M_{3} \\
\alpha_{2} M_{4} \\
\alpha_{3 L} M_{1}+\alpha_{3 R} M_{5} \\
\alpha_{4 L} M_{1}+\alpha_{4 R} M_{6} \\
\alpha_{5} M_{3} \\
\alpha_{6} M_{4}
\end{array}\right] \text {, }} \\
& (\alpha)=\frac{L_{b} /(6 \mathrm{EI})_{b}}{\sum(L / 3 \mathrm{EI})_{\text {elements }}} * \frac{(3 \mathrm{EI} / L)_{\text {coloumn }}}{\sum(3 \mathrm{EI} / L)_{\text {elaments }}-(3 \mathrm{EI} / L)_{b}},
\end{aligned}
$$




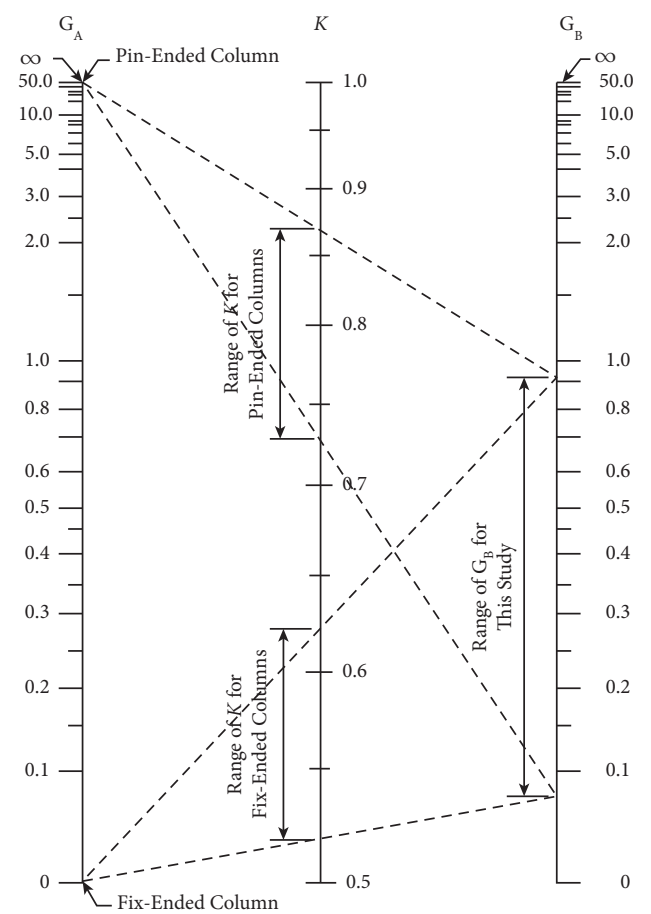

Figure 11: Jackson-Moreland Alignment Chart for braced frames [10].

$L_{b} /(6 \mathrm{EI})_{b}$ is for the connected beam between the studied column and the other column sent the transferred moments, $(3 \mathrm{EI} / L)_{\text {coloumn }}$ is for the studied column, and $(3 \mathrm{EI} / L)_{\text {elements }}$ is for the all connected elements with the studied column including it.

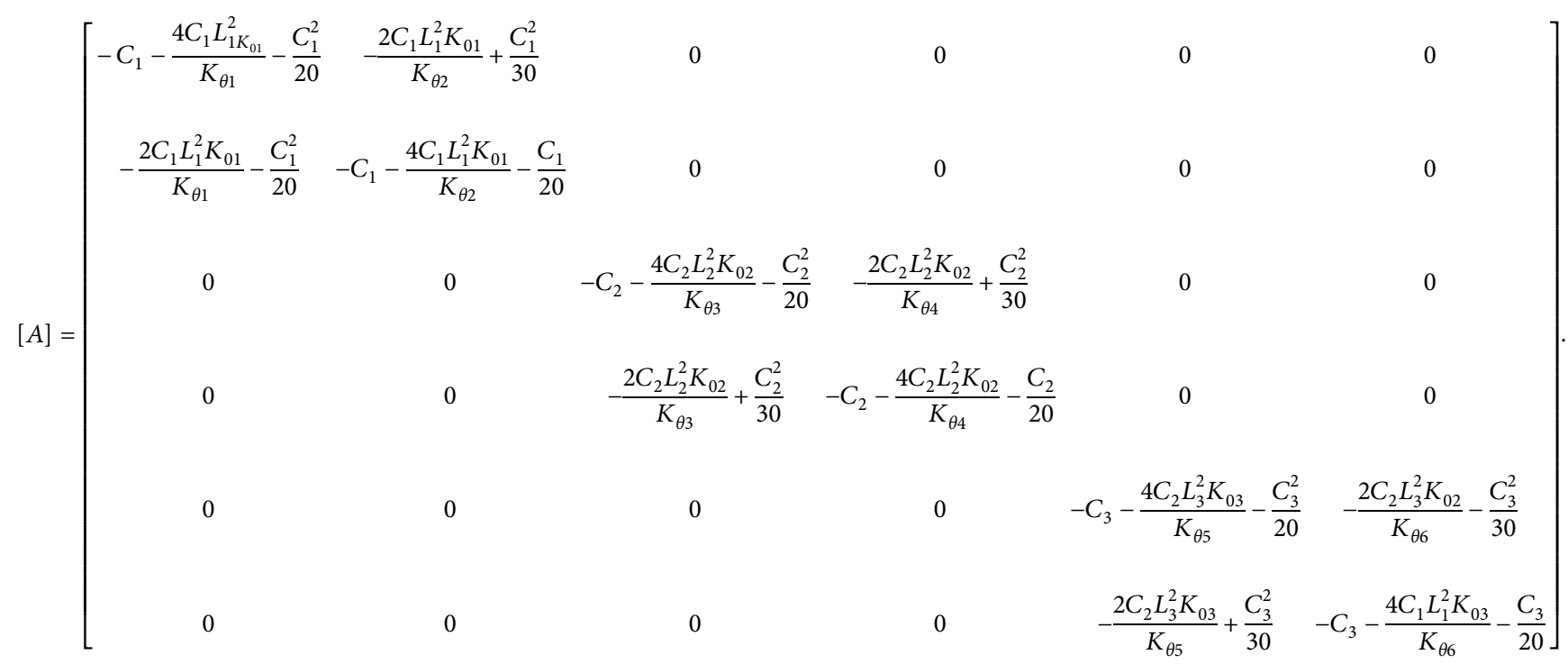

If a number of slender columns exist in the structure and the effect of additional moments of adjacent columns will be considered, the sequence of solving can be schematized as shown in Figure 8(a), or it can be programmed. The shown frame in Figure 8(b) was analyzed by MATLAB program.
M-file of programming and the results are shown in Appendix (I).

7.2. Suggestion 2. In this suggestion, the effect of the additional moments of the adjacent columns on the studied 
column will be taken into the relative rotational stiffness of the connected beams. This will be considered by applying approximate values of additional moments at the ends of the connected beams to evaluate the relative rotational stiffness of these ends for the long columns.

As shown in Figure 10, the initial additional moments can be calculated as follows:

$$
M_{\mathrm{int}}=\frac{w_{e q(a v)}}{4}\left(L-L_{f}\right)^{2}+\frac{w_{e q(a v)}}{4}\left(L-L_{f}\right)
$$

where $w_{e q(a v)}$ is the average equivalent regular load for the second effect due to the end moments of the column, $L_{f}$ : effective length of the long column, and it can be calculated as in Figure 11.

The initial moments of the four joints of the shown frame in Figure 10 will be calculated as follows:

$$
\begin{aligned}
M_{\mathrm{int1}} & =M_{\mathrm{int} 3} \\
& =\frac{p\left(M_{01}+M_{03}\right) L_{c o l}}{8 \mathrm{EI}_{c o l}}\left(L_{c o l}-L_{f}\right), \\
M_{\mathrm{int} 2} & =M_{\mathrm{int} 4} \\
& =\frac{p\left(M_{02}+M_{04}\right) L_{c o l}}{8 \mathrm{EI}_{c o l}}\left(L_{c o l}-L_{f}\right) .
\end{aligned}
$$

And by using the virtual work method, the rotation at the ends of the connected beams at joint 1 as an example can be calculated as follows:

$$
\begin{aligned}
& \theta_{1}=\frac{1}{\mathrm{EI}_{B}}\left(\frac{1}{2} M_{\mathrm{int} 1} L_{b} \cdot \frac{2}{3}+\frac{1}{2} M_{\mathrm{int} 2} \cdot L_{b} \cdot \frac{1}{3}\right), \\
& \theta_{1}=\frac{L_{B}}{\mathrm{EI}_{B}}\left(\frac{M_{\mathrm{int} 1}}{3}+\frac{M_{\mathrm{int} 2}}{6}\right) .
\end{aligned}
$$

The rotational stiffness of the connected beams can be computed as follows:

$$
\begin{aligned}
& K_{\theta 1}=\frac{M_{\mathrm{int} 1}\left(\mathrm{EI}_{b} / L_{b}\right)_{\mathrm{Top}}}{\left(M_{\mathrm{int} 1} / 3+M_{\mathrm{int} 2} / 6\right)}, \\
& K_{\theta 2}=\frac{M_{\mathrm{int} 2}\left(\mathrm{EI}_{b} / L_{b}\right)_{\mathrm{Top}}}{\left(M_{\mathrm{int} 2} / 3+M_{\mathrm{int} 2} / 6\right)}, \\
& K_{\theta 3}=\frac{M_{\mathrm{int} 3}\left(\mathrm{EI}_{b} / L_{b}\right)_{\text {Bottom }}}{\left(M_{\mathrm{int} 3} / 3+M_{\mathrm{int} 4} / 6\right)}, \\
& K_{\theta 4}=\frac{M_{\mathrm{int} 4}\left(\mathrm{EI}_{b} / L_{b}\right)_{\text {Bottom }}}{\left(M_{\mathrm{int} 4} / 3+M_{\mathrm{int} 3} / 6\right)} .
\end{aligned}
$$

And by substituting the computed rotational stiffness in (41), additional moments in the long columns in the closed frame will be computed, taking into account the approximate effect of the additional moments of each column on each other.

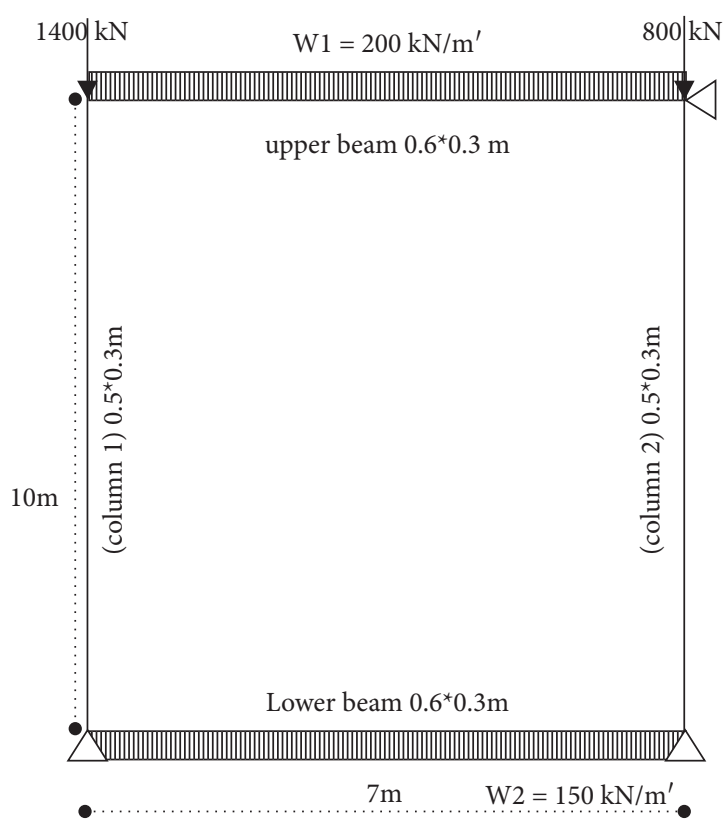

FIgURE 12: The analyzed frame.

\section{Elastic Analysis for Checking the Structural Analysis Efficiency of the Equivalent Column}

Numerous factors will be studied here through linear analysis of closed frames by utilizing the new moment magnifiers matrix and finite element method. The reason for this investigation is to check the structural analysis proficiency of the recommended model in a wide range without the restriction of materials failure. The factors were a variation of the stiffness of connected beams together, induced axial force to Euler load ratio, slenderness ratio, and the stiffness of upper beam to lower beam. The left column is the target column in this study. The results of the comparison are shown in Figures 12- 16.

Solving steps for the equivalent column model:

(1) Calculating the terms $K_{0}, C, K_{\theta 1}$, and $K_{\theta 2}$ according to equations (38-a), (38-b), (48) respectively, to satisfy the moment magnifiers matrix $[A]$.

(2) Applying (41) for each column to compute the additional moments between the columns and the connected beams.

(3) For more accuracy, the effect of the additional moments of each column on each other can be considered by using a schematic method in Figure 8, then find the final additional moments "suggestion 1."

(4) By using model of final loads in Figure 6, the additional moment diagram and additional deformations of the column (lateral displacements and rotations) can be computed.

From the results, it was observed that using (41) in the suggested equivalent column model gives values of additional moments close to their values calculated by the finite 


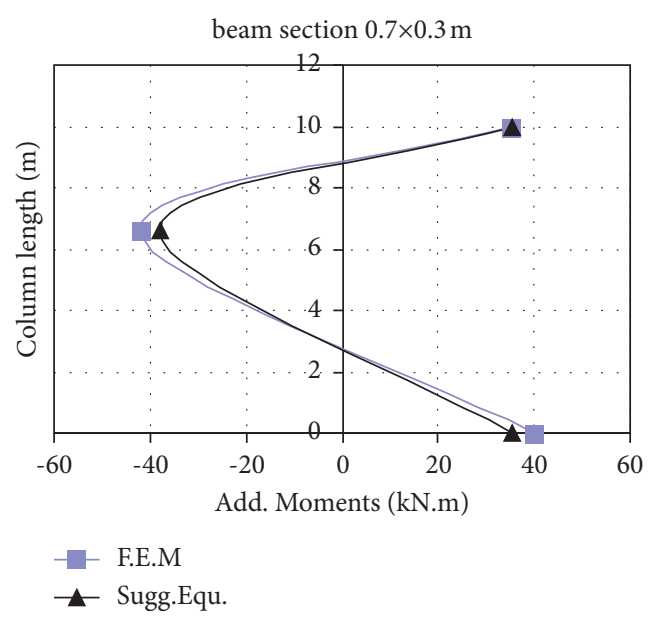

(a)

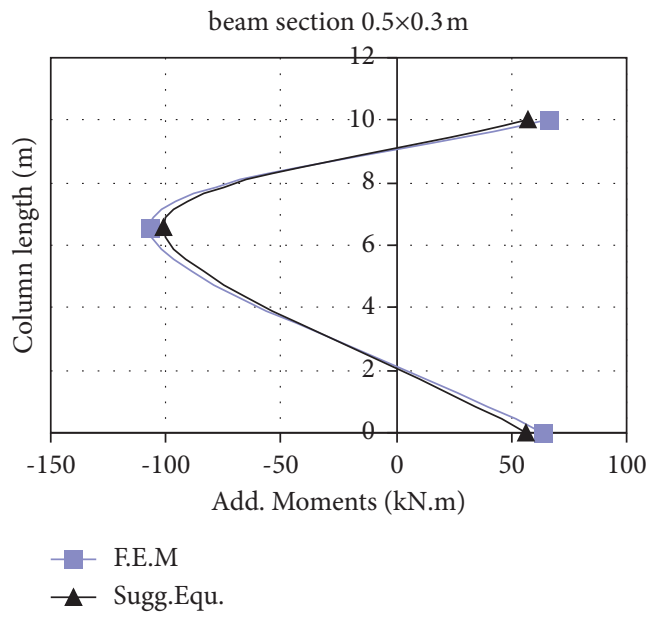

(c)

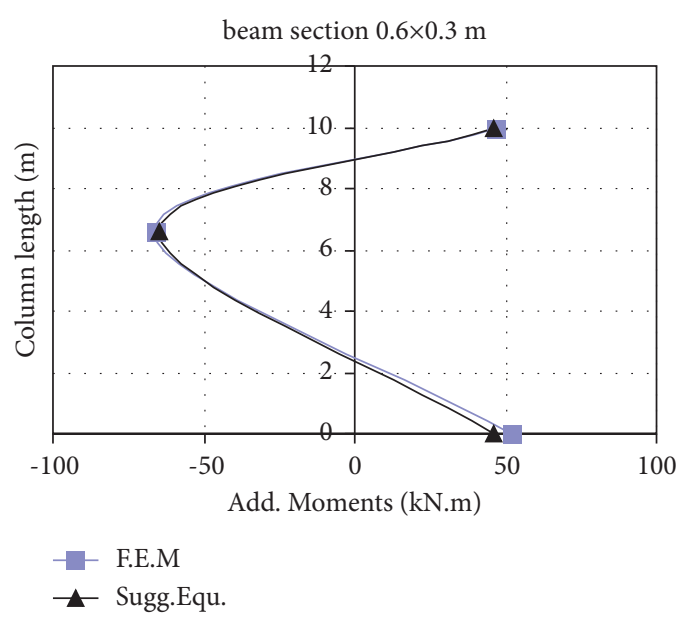

(b)

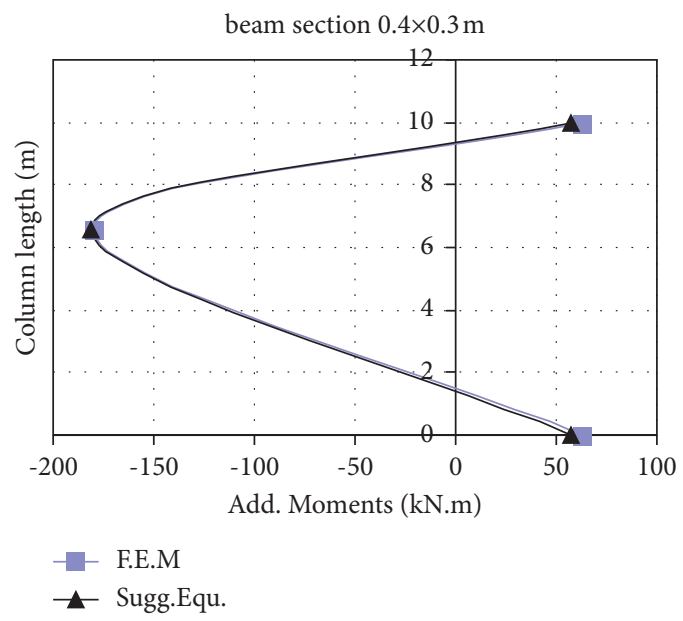

(d)

FIGURE 13: (a-d) Using equivalent column with varying of beam stiffnesses.

element method. The results proved the structural analysis efficiency of the proposed model for analyzing the long column as an isolated element. After the satisfaction to the efficiency of the model, the model can be developed by modifying the flexural rigidity (EI) to appropriate the materials case at the moment of computing the additional moments, as shown in the next section.

\section{Design Procedures for Computing Additional Moments in Long Columns by Using New Moment Magnifiers Matrix}

The calculation of terms $\left(K_{0}, C\right)$ and $\left(K_{\theta 1}, K_{\theta 2}\right)$ in the moment magnifiers matrix of (41) involves the use of the flexural rigidity, EI, of the column and the connected beams, respectively. To use the suggested equation (41) in computing the additional moments in a long column, the flexural rigidity for a given column section must be considered at the time of failure, taking into account the effects of cracking and nonlinearity of the stress-strain curves. James G. MacGregor et al. $[11,12]$ describe empirical attempts to derive values for EI. ACI-318-19 includes two different sets of stiffness values, EI, the first set is for the computation of EI in caudation the critical load of an individual column as follows:

$$
\begin{aligned}
& \mathrm{EI}=\frac{0.2 E_{c} I_{g}+E_{s} I_{s e}}{1+\beta_{d n s}}, \\
& \mathrm{EI}=\frac{0.4 E_{c} I_{g}}{1+\beta_{d n s}},
\end{aligned}
$$

where $E_{c}, E_{s}$ are the modules of elasticity of the concrete and the steel, respectively, $I_{g}$ is the gross moment of inertia of the concrete section, $I_{s e}$ is the moment of inertia of the reinforcement about the centroidal axis of the concrete section, and $\left(1+\beta_{d n s}\right)$ term reflects the effect of creep on the column deflections.

It can be used in equation (57) or equation (58), but equation (57) is more accurate.

The second set is for values of the moment of inertia, I, for use in elastic frame analyses or in computing the effective length factor, $k$. In this set, the column and beam stiffnesses were computed as $0.7 E_{c} I_{c}$ and $0.35 E_{c} I_{c}$, respectively. 


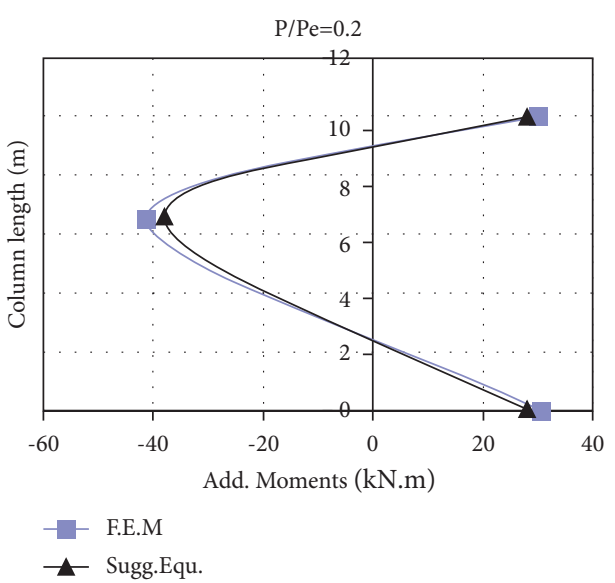

(a)

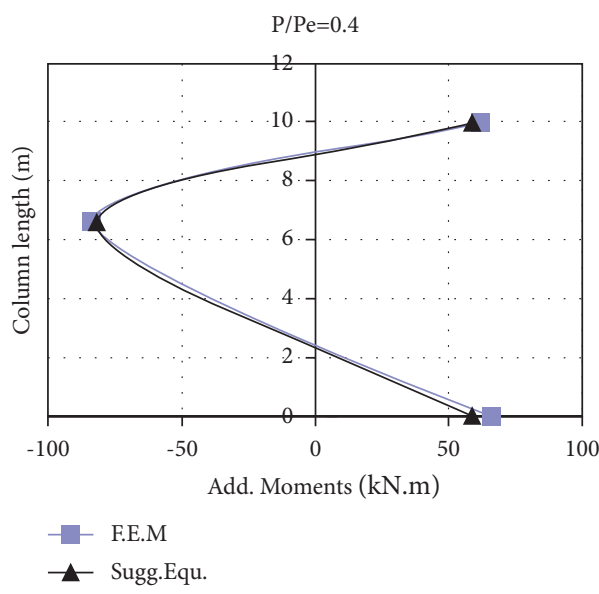

(c)

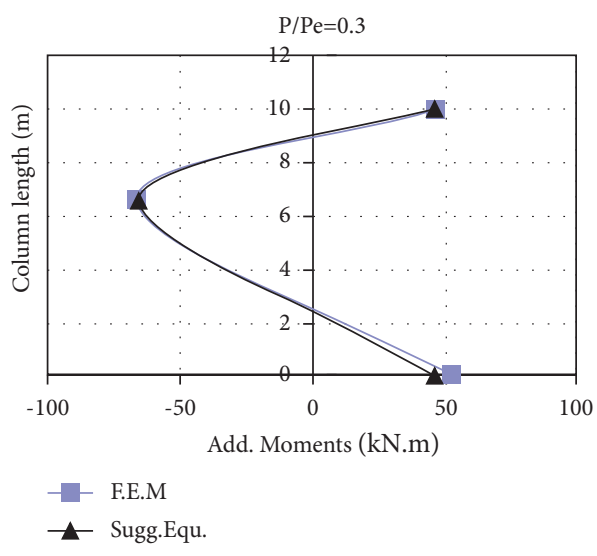

(b)

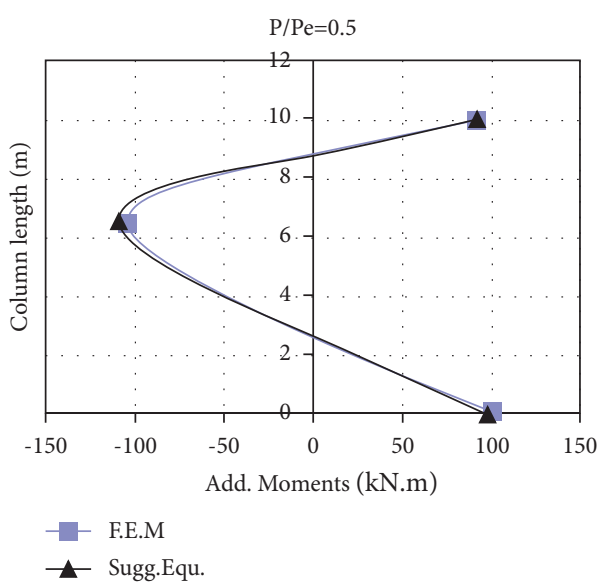

(d)

Figure 14: (a-d) Using equivalent column with varying axial force to Euler load.

According to the concepts of the previous recommendations in ACI-318-19 for using the approximate flexural rigidity in different cases, it is appropriate to use (EI) according to equation (57) or equation (58) when calculating the term (C) in equation (41). Either when calculating the terms $\left(K_{0}\right)$ and $\left(K_{1}, K_{2}\right)$ in $(41)$, the column and beam stiffnesses will be computed as $0.7 E_{c} I_{c}$ and $0.35 E_{c} I_{b}$, respectively.

A long column in closed frames as shown in Figure 10 will be analyzed by each of the equations of (ACI-318-19), (ECP 203-2018), the suggested equation (41) by using suggestions (1), (2) was considered the moment transmitting between the long columns and by using (41)) with neglecting the moment transmitting. The frames were analyzed according to the design requirements and with changing two parameters, slenderness ratio, and $\mathrm{P} / \mathrm{Pe}$. The main cross section of the studied column is $50 \times 30 \mathrm{~cm}$ with a reinforcement ratio in-between 0.01 to 0.035 . The slenderness ratio will be varied from $(9.8-21.5)$ and $\mathrm{P} / \mathrm{Pe}$ will be varied from (0.23-0.95). The relationship between the maximum additional moment through the height of the column and the changed parameters are shown in Figures 18, 17.

The results of the analysis show that there is a large convergence of the additional moments calculated by (41) in the equivalent column between each of suggestions (1) and (2), where the effect of transmitted additional moments was taken into account, and by using equation (41) directly where the transmitted additional moments were neglected. This is expected because of that the failure limitations of the material, which makes the additional moments induced in a long column are not the large values that strongly affect or get affected by the adjacent columns. Thus for easiness, (41) can only be used where a separate analysis of the long column is included without being affected by the additional moments of adjacent long columns.

Moreover, it is observed that (41) gives values of additional moments close to their values which are given by the equation of ACI. This means that using the suggested equation gives good efficiency. Also, it means the appropriate use of flexural rigidity (EI) according to Eqs. (57) or (58) for computing the term (C) in (41) while using the column and beam stiffnesses.

According to $0.7 \mathrm{EI}_{c}$ and $0.35 \mathrm{EI}_{b}$, respectively, for computing the terms $\left(K_{0}\right)$ and $\left(K_{1}, K_{2}\right)$ in (41), the term (C) is the concern of the lateral deformations of the long column, and using (EI) according to Eqs. (57) or (58) in this term corresponds to using these equations for computing Pcr in the equation of ACI. While the terms $\left(K_{0}\right)$ 


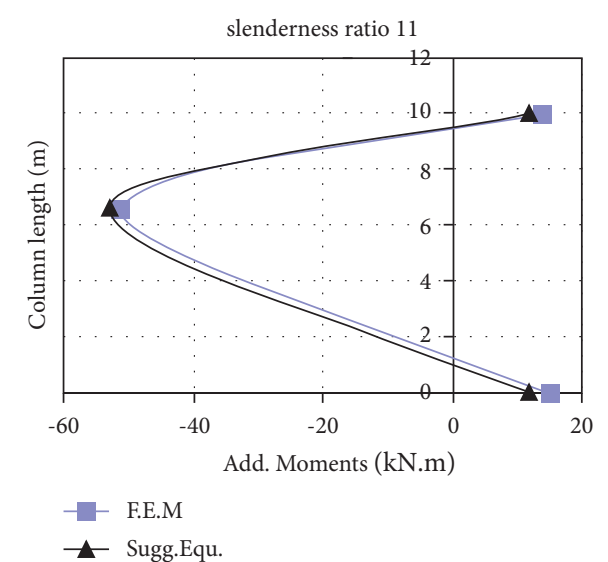

(a)

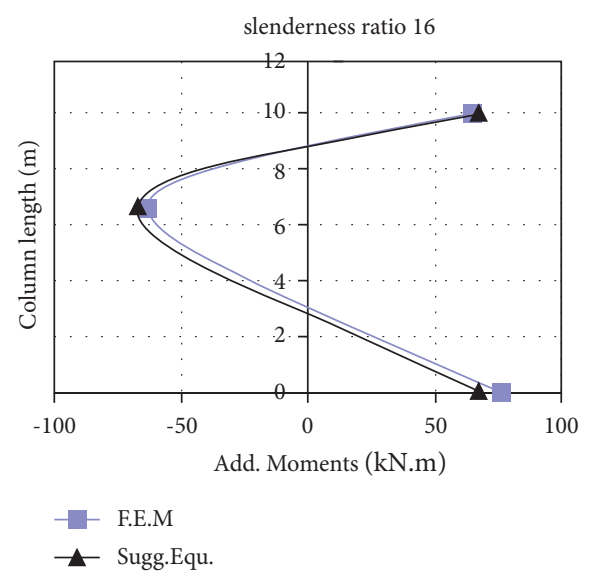

(c)

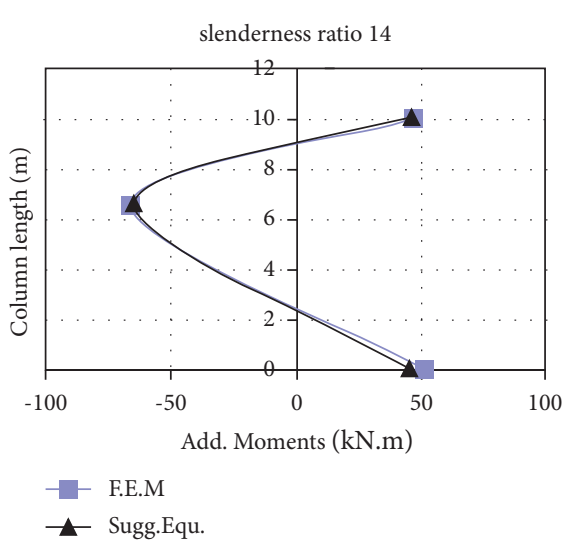

(b)

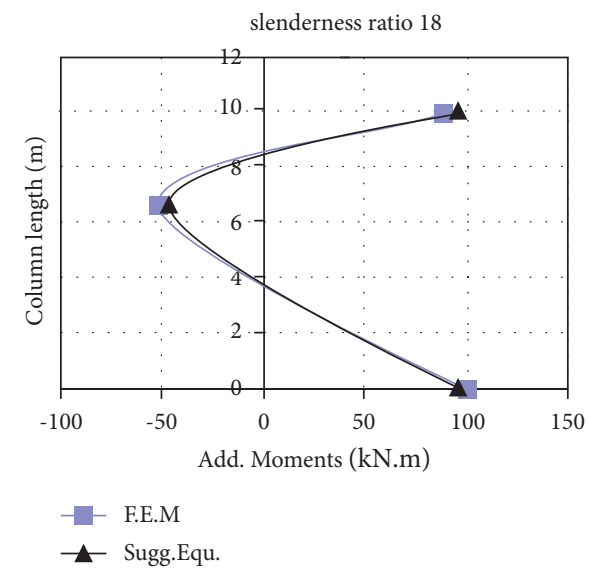

(d)

FIGURE 15: (a-d) Using equivalent column with varying slenderness ratio.

and $\left(K_{1}, K_{2}\right)$ are the concern of the additional moments at the column ends, and the effects of these terms in (41) are similar to the effects of the effective length in the ACI equation.

The additional deformations of the long column (lateral displacement and rotations) at any point can be computed easily, as shown in Figures 19, 20 as an example.

It is clear that there is a big difference in the additional moments between each of ACI, the suggested equation in the equivalent column, and ECP. Prab Bhatt et al. [13] illustrated the basic and the assumptions of computing the additional moments in British code equation BS8110:1997 [14], which is the same as the ECP equation. He illustrates that the deformed column curvature will typically vary along the column as a sinusoidal value of $\left(1 / \pi^{2}\right)$. Figure 21 shows the interaction diagram between the bending and the normal force and strain diagram in the ultimate stage (balanced failure). Thus, the central lateral deflection $a_{u}$ will be assumed as follows.

$$
a_{u}=\left(\frac{1}{\pi^{2}}\right) l_{e}^{2}\left(\frac{1}{r}\right)
$$

The column curvature $(1 / r)$ is calculated based on the strain diagram at the balanced failure, as follows:

$$
\frac{1}{r_{b}}=\frac{(0.003+0.002)}{d}
$$

The maximum deflection for the case set out above is given in the code by the following expression:

$$
\begin{aligned}
a_{u} & =\frac{0.0005 l_{e}^{2}}{h}, \\
a_{u} & =\frac{h}{2000} \cdot\left(\frac{l_{e}}{h}\right)^{2} \\
& =\frac{\lambda^{2}}{2000} \cdot h .
\end{aligned}
$$

It was noted that the ECP equation was based on computing the additional moments in a specific case which is at the balanced failure, and this restriction is difficult to achieve when designing the columns. Thus if the failure mode of the column section is not compatible with the balanced failure, it is supposed that this equation is not valid and it will give far values of the additional acting moments, as shown in previous analyzing cases. So, it seems that the ECP equation cannot be used generally to compute the additional moments and it is for a specific case. Where it can 

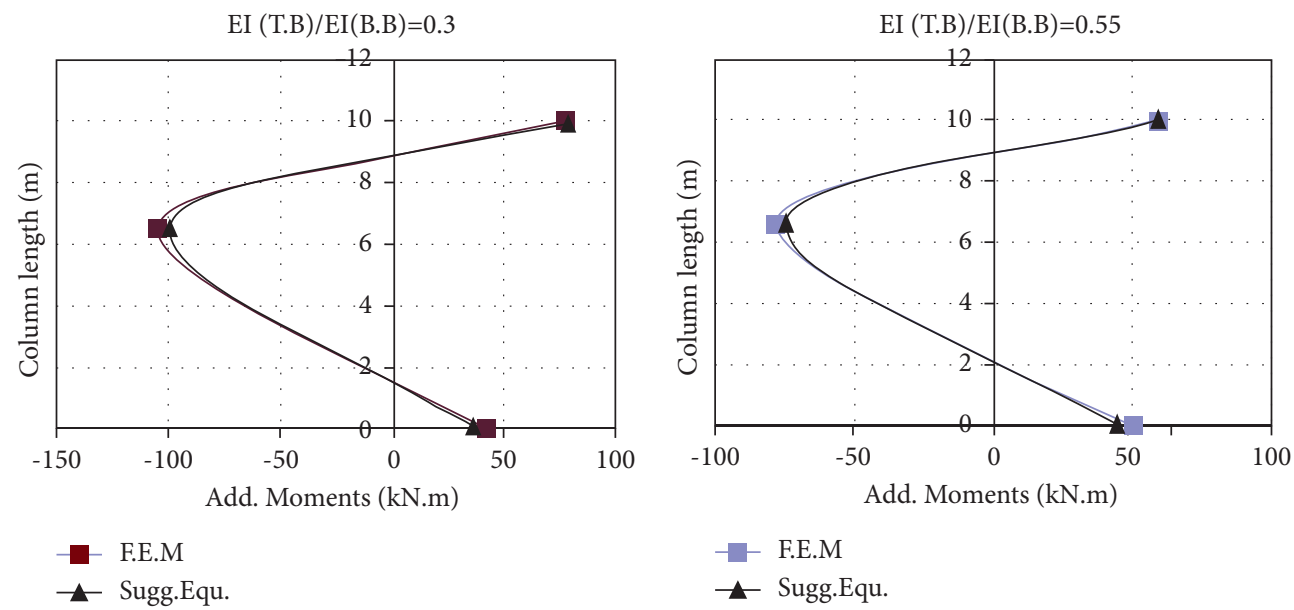

(a)

(b)

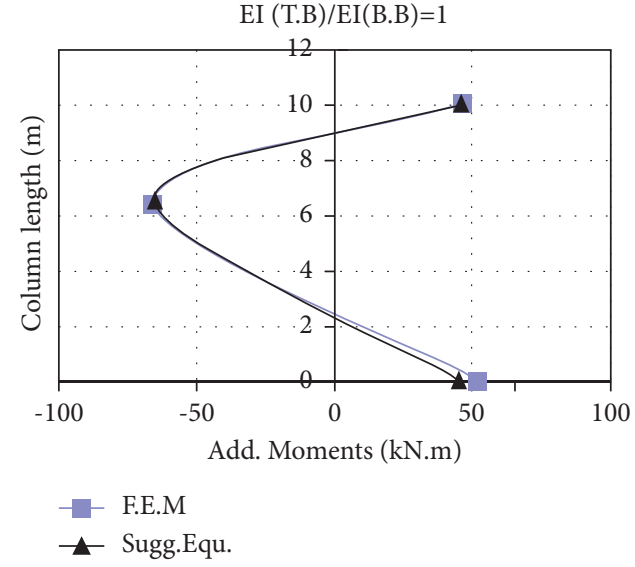

(c)

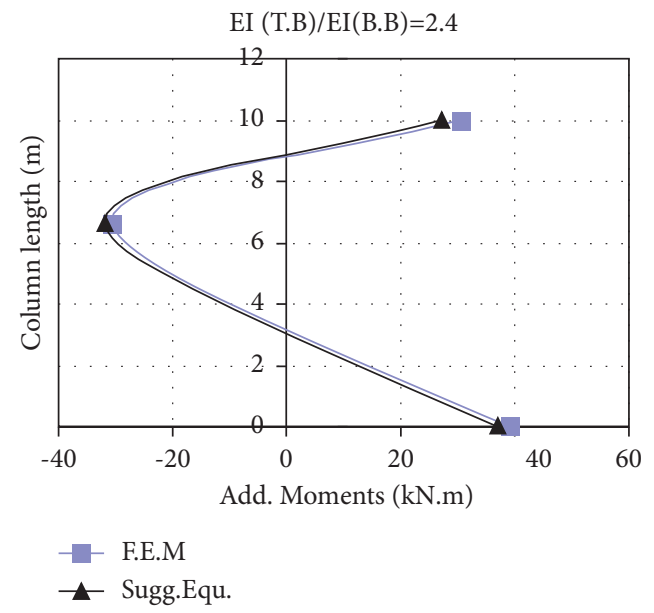

(d)

FigURE 16: (a-d) Using equivalent column with varying upper beam to lower beam stiffness.

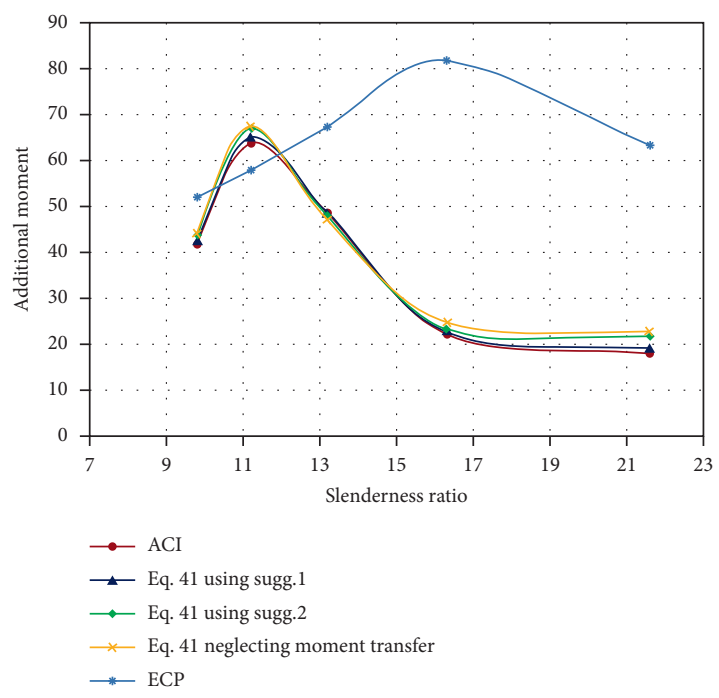

FIGURE 17: Additional moments vs. slenderness ratio.

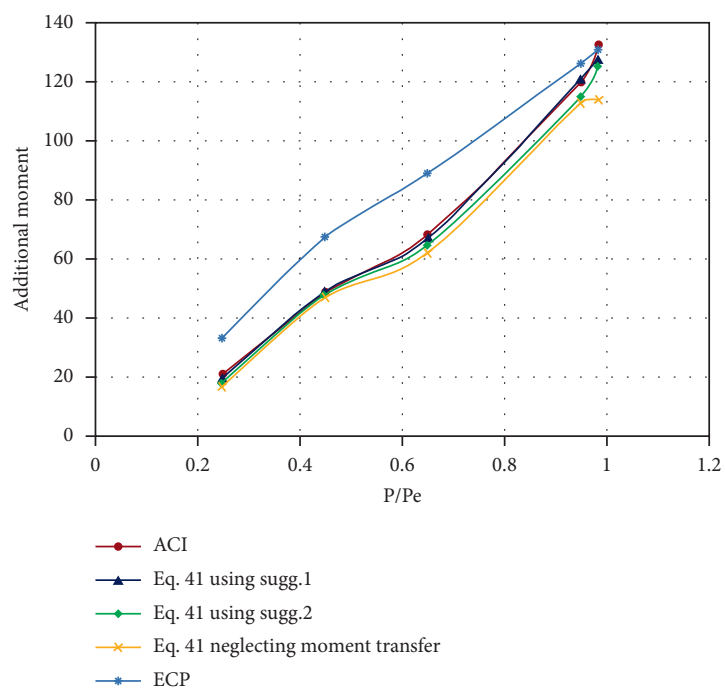

Figure 18: Additional moments Vs. P/Pe. 


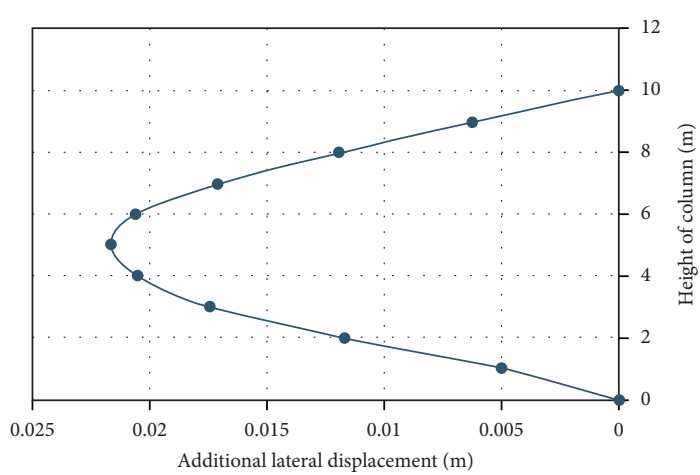

Figure 19: Computing additional lateral displacement by using equivalent column model for slenderness ratio 11.2 as an example.

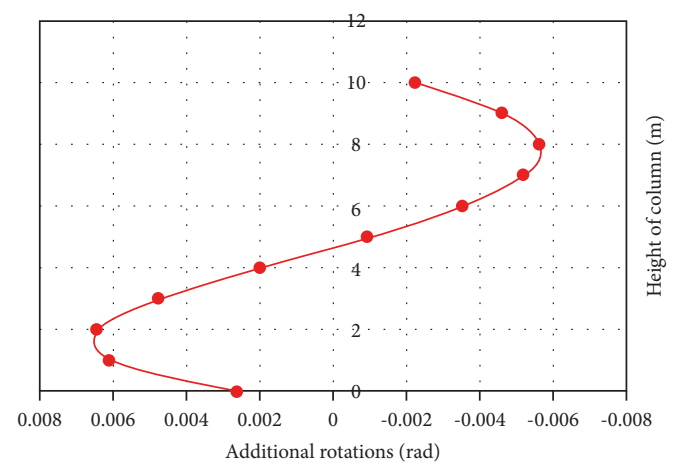

FIgURE 20: Computing the additional rotations by using an equivalent column model for slenderness ratio 11.2 as an example.

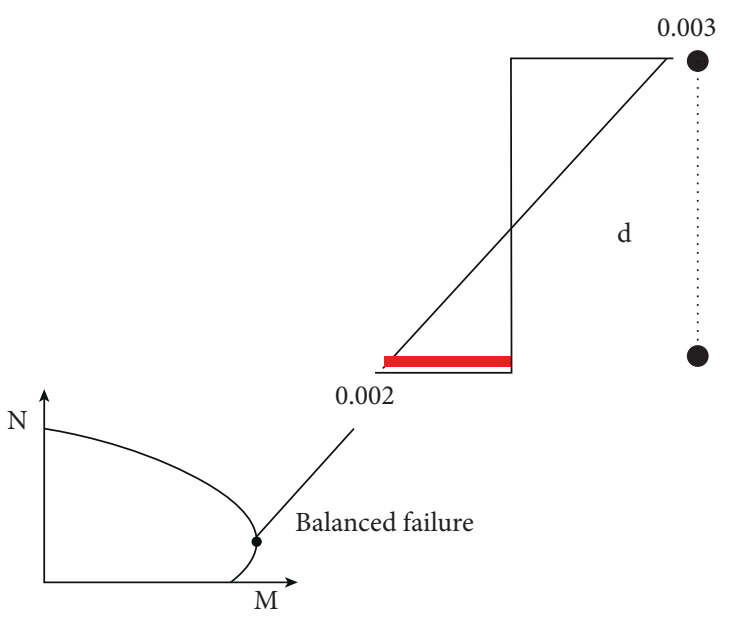

FIGURE 21: The interaction diagram between the bending and the normal force and strain diagram in the ultimate stage (balanced failure).

be used to evaluate the maximum allowable lateral displacement of the column at the balanced failure, then the moment capacity of the column section can be checked for restrained design (balanced failure). This is maybe one of the reasons for the big difference between the results of the ACI equation, the suggested equation in the equivalent column and the EPC equation.
Also, the ECP equation does not take into account the second-order effect which is caused by the axial force and the inverse moments due to beams restriction to the column ends. ECP equation considers only the connected beams effect by dealing with the effective length of the column, not the total length. ACI equation is similar to ECP equation at this point, but returning to the original equation of $\mathrm{ACI}$ (Eq. $62)$, a term $(1+0.23 \mathrm{P} / \mathrm{Pe})$ was found which was omitted from the final equation to generalize the use of the equation, where the factor 0.23 varies as a function of the moment diagram shape. This leads to a decrease in the additional moments among the inflected points, and that approaches the results between the ACI equation and the analysis, which takes into account the second-order effect, which is caused by the inverse moments as in the suggested equivalent column.

$$
M_{c}=\frac{M_{0}\left(1+0.23 P / P_{e}\right)}{1-P / P_{e}} .
$$

On the other hand, the computed flexural rigidity (EI) according to ACI in Eq. (57) remains constant regardless of the magnitude of end moments and, therefore, Pc also remains constant. As a result, the moment magnifier remains constant for a given column. However, Pc is strongly influenced by the effective flexural stiffness (EI), which varies due to the nonlinearity of the concrete stress-strain curve and cracking along the height of the column among other factors [3].

\section{Summary and Conclusions}

Based on the equivalent column concept, a new moment magnifiers matrix was presented in this paper for computing the additional end moments in the braced long column. The equivalent column was an element restricted at its ends by two spring rotational supports and is subjected to lateral distributed loads, which have the same influence of the secondorder effect in a long column. The additional moments' diagram and additional deformations (lateral displacements and rotations) can be computed by using the suggested equivalent column taking into consideration the second-order effect, which is caused by the axial load and the inverse moments due to beams restriction for the column ends, this effect is important although it is neglected in design codes. The long column in the suggested model was analyzed as an isolated element, but by two presented suggestions, the effect of the additional moments of other adjacent long columns, if any, can be considered. The first suggestion took into account the effect of adjacent additional moments by computing the transmitting additional moments among columns through transfer coefficients depended on the rigidity of the connected beams; then the equation of the moment magnifiers matrix was applied more than once for the transmitted moments. This suggestion can be carried out by schematic method as shown in the paper content. In the second suggestion, the adjacent additional moments' effect was considered in the relative rotational stiffness of the connected beams, which are as a rotational spring for the long columns. This will be 
considered by applying approximate values of additional moments at the ends of the connected beams to evaluate the relative rotational stiffness of these beams. Development was carried out on the model by modifying the flexural rigidity (EI) in each of the connected beams and the long column as it is recommended in ACI to appropriate the time of failure. From the results presented in this paper, the following is concluded:

(1) The suggested equivalent column proved a good efficiency for analyzed numerous factors linearly by finite element method, and the equivalent column was satisfying as a successful structural model.

(2) For analyzing many designed long columns in closed frames, the results showed that there are small differences of computed additional moments by applying the suggested equation of moment magnifiers directly and by using the two suggestions of considering the adjacent additional moments, so the additional moments of the adjacent columns can be neglected for simplifying.

(3) The developed model gave close values of the additional moments for many analyzed long columns with ACI equation, and it is appropriate to generalize this model for second-order analysis of long braced columns as an easy-to-use model that yields good results.

(4) There was a gap between the values of the additional moments computed by the two methods, the suggested equivalent column and ECP equation. This may be because ECP equation was based on computing the additional moments in a specific case which is at the balanced failure. Moreover, the ECP equation did not take into account the second-order effect, which is caused by the axial force and the inverse moments due to beams' restriction for the column ends.

\section{Appendix}

M-File in MATLAB program to solve the frame in Figure 8(b).

The results in Command window of MATLAB program.

\section{Data Availability}

The data used to support the findings of this study are included within the article and are available from the corresponding author upon reasonable request.

\section{Conflicts of Interest}

All authors declare that they have no conflicts of interest. by the Deanship of Scientific Research at Jouf University under grant No (DSR-2021-02-0353).

\section{References}

[1] J. G. Wight and J. K. MacGregor, Reinforced concrete: Mechanics and Design, Prentice-Hall, Upper Saddle River, NJ, USA, 6th ed. edition, 2011.

[2] R. D. Cook, D. S. Malkus, and M. E. Plesha, Concepts and Applications of Finite Element Analysis, John Wiley \& Sons, Hoboken, NJ, USA, 3rd edition, Article ID 0-471-84788-7, 1989.

[3] T. K. Tikka and S. A. Mirza, "Effective length of reinforced concrete columns in braced frames," International Journal of Concrete Structures and Materials, vol. 8, no. 2, pp. 99-116, 2014.

[4] Aci Committee 318, Building Code Requirements for Structural concrete (ACI 318-19) and Commentary, American Concrete Institute, Farmington Hills, MI, USA, 2019.

[5] Csa, Design of concrete Structures, Vol. A23.3-04, Canadian Standards Association, Mississauga, Canada, 2004.

[6] W. F. Chen and E. M. Lui, Structural Stability-Theory and Implementation, Elsevier Science Publishing Company Inc, New York, NY, USA, 1987.

[7] EN 1992-1-1, Eurocode 2 (EC2): Design of concrete StructuresPart 1-1: General Rules and Rules for buildings, European Committee for Standardization, Brussels, Belgium, 2004.

[8] Ecp, Egyptian Code for Design and Construction of Reinforced Concrete Structures, ECP, Tamworth, UK, 2018.

[9] H. M. Afefy and E.-T. M. El-Tony, "Simplified design procedure for reinforced concrete columns based on equivalent column concept," International Journal of Concrete Structures and Materials, vol. 10, no. 3, pp. 393-406, 2016.

[10] L. Duan, W. S. King, and W. F. Chen, "K-factor equation to alignment charts for column design," ACI Structural Journal, vol. 90, no. 3, pp. 242-248, 1993.

[11] J. G. MacGregor, U. H. Oelhafen, and S. E. Hage, "A Reexamination of the El Value for Slender Columns" Reinforced Concrete Columns, pp. 1-40, American Concrete Institute, Farmington Hills, MI, USA, 1975.

[12] J. G. MacGregor, J. E. Breen, and E. O. Pfrang, "Design of slender columns," ACI Journal, Proceedings, vol. 67, no. 1, pp. 6-28, 1970.

[13] P. Bhatt, J. T. MacGinly, and B. S. Choo, "Reinforced Concrete, Design Theory and Examples", CRC Press, Boca Raton, FL, USA, 3rd edition, 2006.

[14] Bs8110, Structural Use of Concrete Part 1: Code of Practice for Design and Construction, BSI, London, UK, 1997.

\section{Acknowledgments}

The authors extend their appreciation to the Deanship of

Scientific Research at Jouf University. This work was funded 